Published in final edited form as:

Kumru, B., Molinari, V., Dünnebacke, R., Blank, K. G., \& Schmidt, B. V. K. J. (2019). Extremely Compressible Hydrogel via Incorporation of Modified Graphitic Carbon Nitride.

Macromolecular Rapid Communications, 40(4): 1800712. doi:10.1002/marc.201800712.

\title{
Extremely Compressible Hydrogel via Incorporation of Modified Graphitic Carbon Nitride
}

\author{
Baris Kumru, Valerio Molinari, Reinhild Dünnebacke, Kerstin G. \\ Blank, Bernhard V. K. J. Schmidt
}

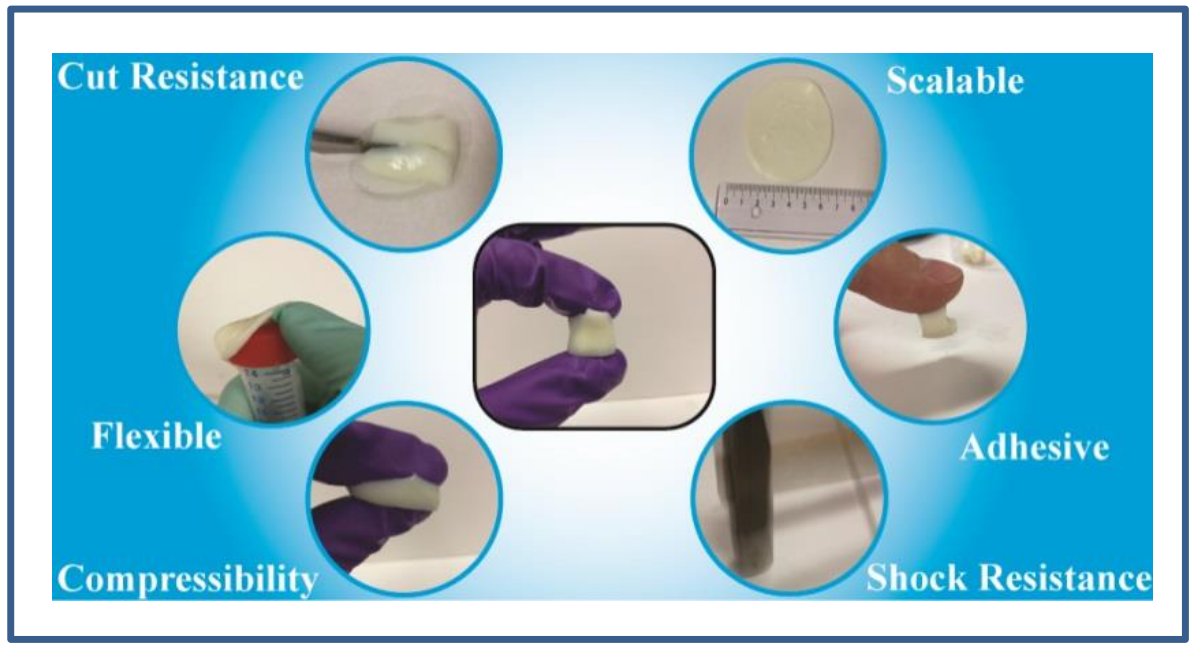

Surface functionalized Carbon Nitride facilitates the formation of extremely compressible hydrogels that withstand forces up to $12 \mathrm{MPa}$. In addition, the hydrogels feature tissue adhesive properties, shock resistance, cut resistance and little to no toxicity. 
Extremely Compressible Hydrogel via Incorporation of Modified Graphitic Carbon Nitride

Baris Kumru, ${ }^{a}$ Valerio Molinari, ${ }^{a}$ Reinhild Dünnebacke, ${ }^{b}$ Kerstin G. Blank, ${ }^{* b}$ Bernhard V. K. J. Schmidt $* a$

Dr. Bernhard V.K.J. Schmidt

${ }^{a}$ Max-Planck-Institute of Colloids and Interfaces; Department of Colloid Chemistry, Am

Mühlenberg 1, 14476 Potsdam, Germany

E-mail: bernhard.schmidt@mpikg.mpg.de

Dr. Kerstin G. Blank

${ }^{\mathrm{b}}$ Max-Planck-Institute of Colloids and Interfaces; Mechano(bio)chemistry, Am Mühlenberg 1, 14476 Potsdam, Germany

E-mail: kerstin.blank@mpikg.mpg.de

Keywords: ((hydrogels, carbon nitride, photoinitiation, compressible))

Extremely compressible hydrogels are fabricated in one pot via sulfonic acid modified graphitic carbon nitride (g-CN-AHPA) as visible light photoinitiator and reinforcer. The hydrogels show unusual compressibility upon applied stress up to $12 \mathrm{MPa}$, presenting temporary physical deformation and remain undamaged after stress removal despite their high water content $(90 \mathrm{wt} . \%)$. Cyclic compressibility proves the fatigue resistance of the covalently and electrostatically reinforced system that possesses tissue adhesive properties, shock resistance, cut resistance and little to no toxicity.

Hydrogels are crosslinked materials that absorb significant amounts of water and possess mechanical features similar to biological systems, such as soft tissue and cartilage. ${ }^{[1]}$ Being weak under regular synthesis conditions, significant effort was invested in reinforcing hydrogels to increase their mechanical properties and to mimic natural systems. ${ }^{[2]}$ Common approaches for reinforcement utilize double network systems, ${ }^{[3-5]}$ nano composite hydrogels, ${ }^{[6-}$ ${ }^{8]}$ host-guest systems, ${ }^{\left[{ }^{[9-10]}\right.}$ and solid reinforcer additions. ${ }^{[11-13]}$ Reinforcers act as a stress absorbing material and ideally distribute the stress equally through the polymeric network. From a chemical perspective, reinforcement can be achieved via covalent bonding, ionic or $\pi$ $\pi$ interactions between reinforcer and polymer network. ${ }^{[14]}$ Even though covalently reinforced materials may show high compression strength, covalent bonding causes low fatigue resistance and no self-healing as it is not possible to recover the damaged bond. Aforementioned reasons led researchers to investigate more complex systems, e. g. via ionic interactions, to create materials with high compressibility with a drawback of less water content or usage of high-value chemicals. ${ }^{[15-16]}$ Interpenetrating networks and supramolecular reinforcements are well-known to provide excellent mechanical properties. However, their drawbacks are tedious synthesis procedures and insufficient water content, which is an important factor for possible bioapplications. Metal coordinated reinforcements provide significant compressibility yet still have water deficiencies. On the other hand, creative ways of covalent reinforcement might lead to novel materials with exceptional properties. Design of a covalently reinforced hydrogel, which does not break under compression at all, would eliminate the doubts regarding negative perspectives of covalent reinforcement.

Graphitic carbon nitride $(\mathrm{g}-\mathrm{CN})$ is a metal-free visible light active photocatalyst with tunable properties, which is composed of repeating tri-s-triazine units. ${ }^{[17-18]}$ It is synthesized from low cost precursors and possesses high activity in applications such as water splitting, ${ }^{[19]}$ 
$\mathrm{CO}_{2}$ reduction, ${ }^{[20]}$ emulsion stabilization ${ }^{[21]}$ and designed organic reactions. ${ }^{[22-23]}$ Reversible assembly of g-CN particles in water was shown to provide hydrogel-like 3D networks, presenting dye absorption and degradation platforms. ${ }^{[2]}$ Recently, it was employed in polymer chemistry to perform photopolymerization ${ }^{[25-26]}$ or fabricate hydrogels. ${ }^{[27-31]}$ It was shown that g-CN sheets act as reinforcer and photoinitiator in radical mediated hydrogel formation with surface charge being one of the important parameters regarding hydrogel strength. ${ }^{[27]}$ Our group recently developed an one pot grafting method to anchor functional groups on the surface of g-CN, showing excellent dispersions in aqueous and organic media. ${ }^{[32]}$ In such a way, 3-allyloxy-2-hydroxy-1-propanesulfonic acid (AHPA) groups can be grafted onto the g-CN surface (g-CN-AHPA) for enhanced dispersibility in water and lower surface zeta potential, which may lead to the formation of strong hydrogels.

Herein, novel hydrogel materials with outstanding compressibility, cut and shock resistance as well as tissue adhesive properties with moderate flexibility and little to no toxicity are reported. The hydrogels are based on g-CN-AHPA as reinforcer and initiator, activated via visible light irradiation (Scheme 1). Common reinforcements generally target tough and stiff hydrogels with strong mechanical properties. However, a novel type of reinforcement is introduced here, which enables to synthesize soft hydrogels with outstanding mechanical performance as well as tuned mechanical properties via variation of the $\mathrm{g}$-CNAHPA content. Overall, an easy approach is presented to fabricate extremely compressible hydrogels in one step via visible light and encompassing high water contents around $90 \%$.

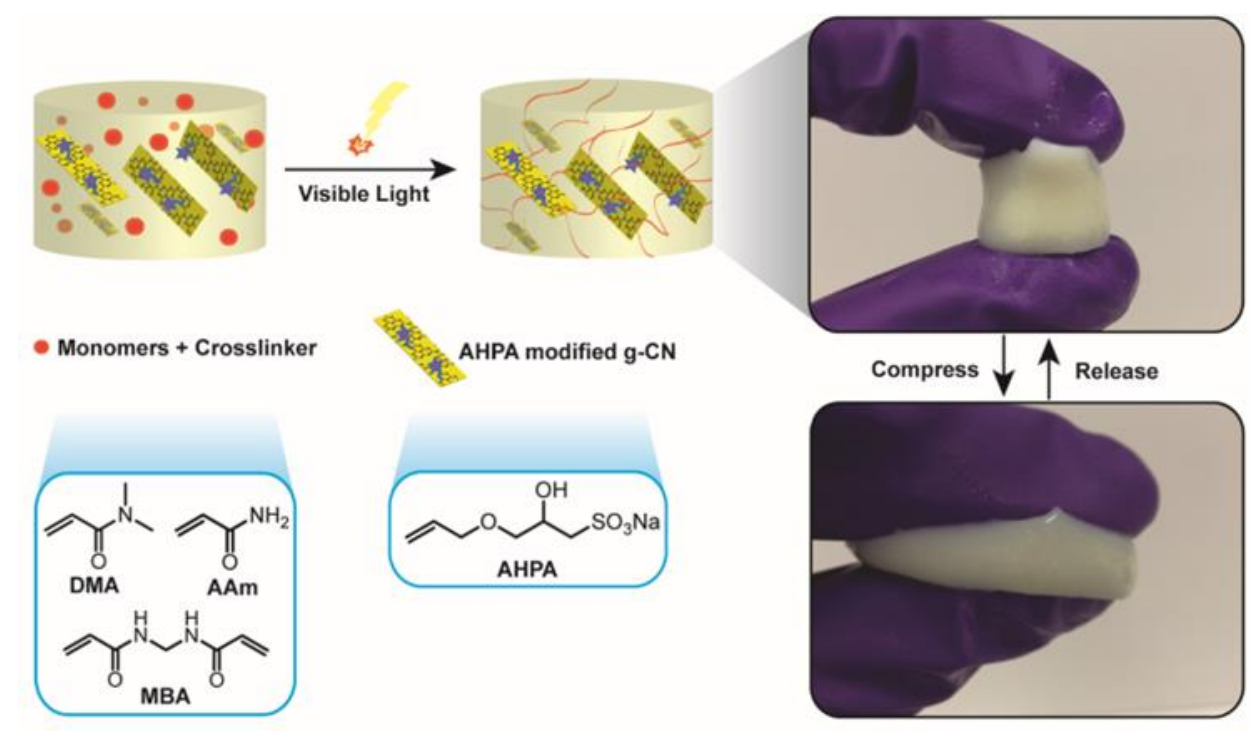

Scheme 1. Overview for the synthesis of g-CN-AHPA hydrogel and images of final hydrogel product.

Sulfonic acid functionalized g-CN (g-CN-AHPA) was synthesized via cyanuric acidmelamine complex and surface modification as reported in literature. ${ }^{[32-33]}$ Modification of $\mathrm{g}$ CN with AHPA was confirmed via elemental analysis and zeta potential (Table S1, Supporting Information), as well as transmission electron microscopy (Figure S1, Supporting Information), energy-dispersive C-ray spectroscopy mapping in scanning electron microscopy (SEM) (Figure S2, Supporting Information) and spectroscopy (Figure S3, Supporting Information). In the next step, different concentrations of g-CN-AHPA were employed for one pot, visible light induced synthesis of hydrogels with acrylamide (AAm)/ $\mathrm{N}, \mathrm{N}$ dimethylacrylamide (DMA) monomer mixtures (3:1) with $N, N^{\prime}$-methylenebis(acrylamide) (MBA) crosslinker in water (Table 1, details in Supporting Information). Hydrogels with water contents between $87 \%$ and $92 \%$ were synthesized and characterized via rheology. 
Table 1. Overview of mechanical properties of g-CN-AHPA hydrogels.

\begin{tabular}{|c|c|c|c|c|c|c|}
\hline $\begin{array}{l}\text { g-CN-AHPA } \\
\text { content } \\
\text { (wt.\%) }\end{array}$ & $\begin{array}{l}\text { Water } \\
\text { content } \\
\text { (wt. \%) }\end{array}$ & $G^{\prime}(\mathbf{P a})^{\mathrm{c}}$ & $G^{\prime \prime}(\mathrm{Pa})^{\mathrm{c}}$ & $G^{\prime}(\mathrm{Pa})^{\mathrm{d}}$ & $\begin{array}{l}\text { Change in } G \\
\text { after back } \\
\text { process }(\%)^{c}\end{array}$ & $E_{\bmod }(\mathrm{MPa})^{\mathrm{e}}$ \\
\hline $0.35^{\mathrm{a}}$ & 99 & 469 & 69.4 & 387 & -13 & 0.41 \\
\hline $1^{\mathrm{b}}$ & 91 & 584 & 130 & 645 & 15 & 0.92 \\
\hline $2^{\mathrm{b}}$ & 90 & 114 & 44.1 & 123 & 14 & 1.63 \\
\hline $3.5^{\mathrm{b}}$ & 89 & 5840 & 1300 & 6450 & 17 & 10.76 \\
\hline $5^{\mathrm{b}}$ & 87 & 44300 & 3940 & 27100 & -42 & 0.21 \\
\hline
\end{tabular}

${ }^{\mathrm{a}}$ composed of $0.45 \mathrm{wt} . \%$ AAm, 0.15 wt. $\%$ DMA and $0.05 \mathrm{wt} . \% \mathrm{MBA},{ }^{\mathrm{b}}$ composed of $6 \mathrm{wt} . \%$ AAm, 2 wt. $\%$ DMA and 0.03 wt. $\% \mathrm{MBA}^{\mathrm{c}}$ at $0.1 \%$ strain, ${ }^{\mathrm{d}}$ at $20 \%$ strain, ${ }^{\mathrm{e}}$ calculated at $10 \%$ strain before the break or at $10 \%$ before maximum strain.

The formed materials are in gel state at any strain (storage moduli $\left(G^{\prime}\right)>$ loss moduli $(G$ ' $)$ ) and $G$ 'values show a slight increase with increased frequency (Figure S4, Supproting Information). Materials show a decrease in $G^{\prime}$ values with increasing strain due to shear thinning effect, which is more pronounced with high g-CN-AHPA content (5 wt.\%, 38\% decrease in G' value at $20 \%$ strain). The softest hydrogel with the lowest $G$ ' value is $2 \mathrm{wt} . \%$ g-CN-AHPA hydrogel, however it shows excellent recovery in $G$ ' and $G$ ', values at any strain (Figure 1a). Upon rotational compression in the rheometer, the self-standing $2 \mathrm{wt} \% \mathrm{~g}-\mathrm{CN}$ AHPA hydrogel shows an outstanding compressibility, remaining undamaged after removal of stress (Video S1, Supporting Information).

Solid state analysis shows the incorporation of g-CN-AHPA into the hydrogels (Figure S5, supporting Information), e.g. UV-Vis spectra of freeze dried hydrogels follow the characteristic absorption of g-CN-AHPA (220-480 nm). XRD shows signal around $27^{\circ}$, which is assigned to the typical aromatic interlayer stacking of g-CN sheets and the intensity correlates with the incorporated amount of g-CN-AHPA. In addition, FT-IR results show bands from both g-CN (bands between $1630-1230 \mathrm{~cm}^{-1}$ from $\mathrm{C}=\mathrm{N}$ and $\mathrm{C}-\mathrm{N}$ stretching) and monomers (around 1750 and $2900 \mathrm{~cm}^{-1}$ as carbonyl and $\mathrm{N}-\mathrm{H}$ stretching). SEM image of freeze dried 2 wt.\% g-CN-AHPA and relative elemental mapping clearly shows the incorporation of sulfur atoms arising from sulfonic acid groups of g-CN-AHPA, which is homogenously distributed through the porous hydrogel network (Figure S6, Supporting Information).

Subsequently, compression tests were performed for more information on the mechanical properties of hydrogels. Interestingly, 0.35 wt. $\%, 1$ wt. $\%$ and 5 wt. $\%$ g-CNAHPA containing hydrogels show mechanical failure at low forces $(<1 \mathrm{MPa})$ and resulted in cracking under compression (Figure 1b). The intermediate hydrogel with 3.5 wt. $\%$ g-CNAHPA shows resistance up to $12 \mathrm{MPa}$ but breaks at a strain around 50\%. Remarkably, 2 wt.\% g-CN-AHPA containing hydrogel showed excellent compressibility even at forces of $10 \mathrm{MPa}$. The soft material shows almost no resistance to compression up to $70 \%$ strain but shows significant response at high strain values $(10 \mathrm{MPa})$, remaining undamaged after removal of stress (Figure 1b). The cylindrical shaped material shows remarkable elastic properties as it transforms into a thin and flat form upon strong compression. The original shape is immediately retained after the removal of stress (Figure 1e), even when the stress is not equally received (Video S2, Supporting Information). Herein, another important factor to discuss is absorption of the applied stress. In most of the known hydrogel systems non- 
uniform stress causes dramatic damage via unequal stress distribution, which is in contrast to the presented g-CN-AHPA hydrogel. Elastic modulus values showed quite low results for $0.35,1$ and 5 wt.\% g-CN-AHPA hydrogels, while 2 wt. $\%$ and 3.5 wt. $\%$ g-CN-AHPA hydrogels possess the highest elastic modulus values (Table 1). These calculations were performed at $10 \%$ strain before the break or at $10 \%$ before maximum strain. However, 2 and $3.5 \mathrm{wt} . \% \mathrm{~g}$-CN-AHPA hydrogels possess different compression profiles as they show a nongradual increase. Therefore, elastic moduli values were also calculated right before the break, which follow the same trend as 10\% before the break. Especially 2 and $3.5 \mathrm{wt} . \% \mathrm{~g}-\mathrm{CN}$-AHPA hydrogels show very high elastic moduli (64.1 and $68.7 \mathrm{MPa}$, respectively) (Table S2, Supporting Information). $2 \mathrm{wt} . \% \mathrm{~g}$-CN-AHPA hydrogel is flexible enough under compression and can withstand stress up to $2 \mathrm{MPa}$, even when the force is received non-uniformly. Due to physical changes upon compression (regardless of initial shape and surface area), a rough calculation of the compressive strength value for the presented hydrogel yields approximately $10 \mathrm{MPa}$, which is remarkable for a covalent hydrogel, especially when compared to systems with non-covalent reinforcements and having the high water content of the presented hydrogel in mind (Table S3, Supporting Information). ${ }^{[4,6,15,16,34]}$ The mechanical performance of the hydrogel is clearly in the range to meet the criteria for tough cartilage-joint systems where the average cyclic stress ranges from $6 \mathrm{MPa}$ up to $14 \mathrm{MPa}$ with daily activities like walking or running. ${ }^{[35,36]}$ One important, distinct characteristic of the presented hydrogel lies beneath its softness, which allows improved stress load distribution compared to though hydrogels.

Cyclic compression of $2 \mathrm{wt} \% \mathrm{~g}$-CN-AHPA hydrogel at high forces shows recovery of the material properties, which proves the durability of the hydrogel system (Figure 1d). Durability of a material containing a covalently bonded reinforcer is an important factor, as possible damages are non-healable. The 2 wt.\% g-CN-AHPA hydrogel shows excellent performance at $2 \mathrm{MPa}$ of applied stress, going from completely thin and flat structure back to original shape for at least 50 cycles (Video S3, Supporting Information), which is a clear statement for the fatigue resistance of the material.
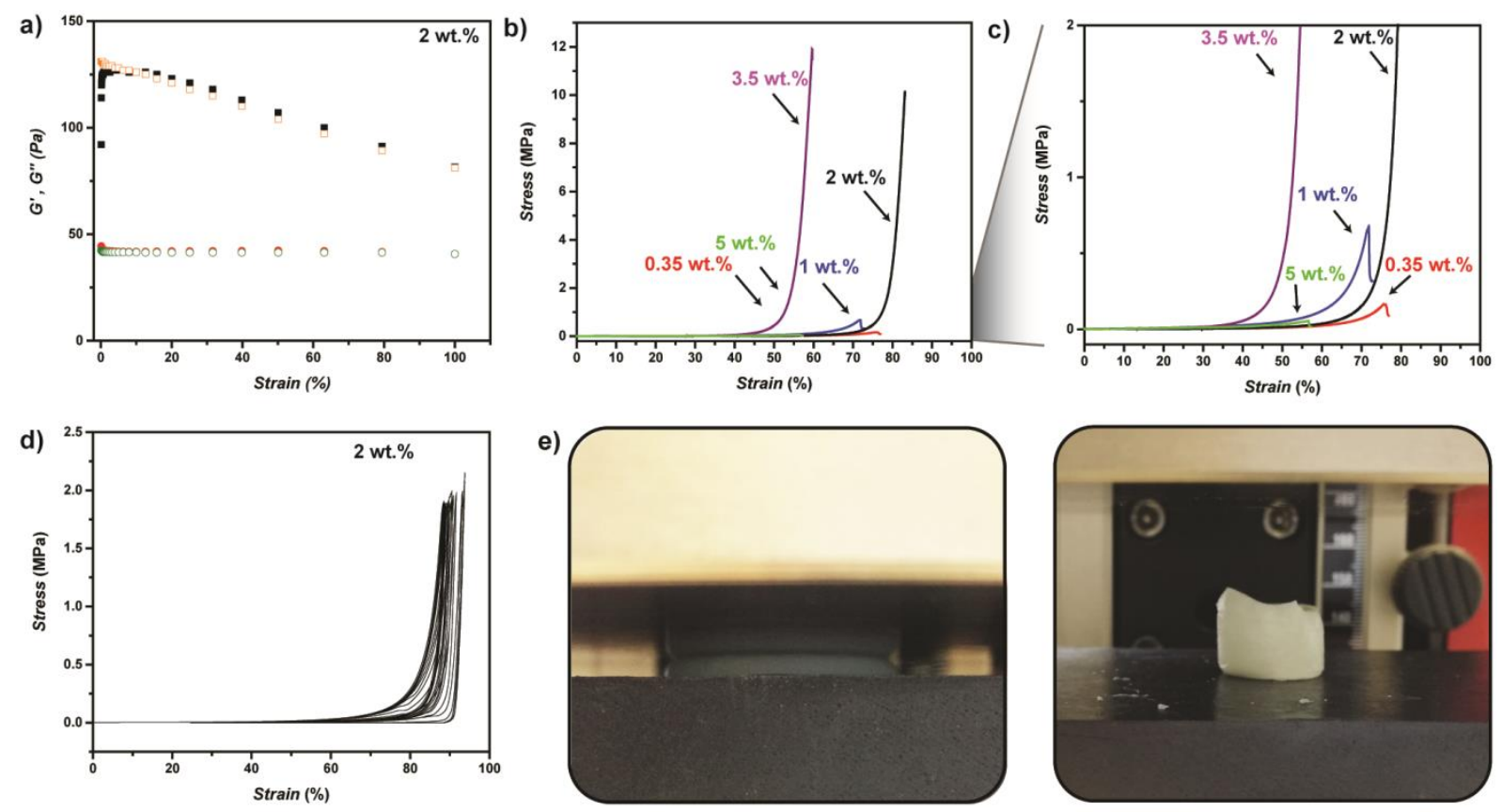

Figure 1. a) Rheology results of 2 wt.\% g-CN-AHPA hydrogel (black squares: $G$ ' forth process; orange squares: $G$ ' back process; red circles: $G$ ' forth process; green circles: $G$ ' back process), b) Compression test results of g-CN-AHPA hydrogels (red: 0.35 wt.\%; blue: 1 
wt.\%; black: 2 wt.\%; violet: 3.5 wt.\%; green: 5 wt.\% ), c) magnification of compression test results of g-CN-AHPA hydrogels, d) cyclic compression (50 cycles) test results of $2 \mathrm{wt} \% \mathrm{~g}$ CN-AHPA hydrogel and e) images of 2 wt.\% g-CN-AHPA hydrogel during (left) and after (right) compression.

2 wt.\% g-CN-AHPA hydrogel has other interesting properties as well, e.g. it is quite resistant against compression with a scalpel (Figure 2a), remaining undamaged even after repetitive compressions (Video S4, Supporting Information). For such a soft hydrogel with covalent as well as electrostatic reinforcement, resistance to compression with sharp materials shows the strength of the network. In addition, the hydrogel is also flexible enough to cover surfaces such as polypropylene caps (Figure 2b) or bending the circular shaped hydrogel around a finger (Video S5, Supporting Information).

In order to elucidate future applications in the biomedical field, first investigations regarding toxicity of the present hydrogels were conducted as toxic effects might arise from residual unreacted monomer moieties or the incorporation of $\mathrm{g}-\mathrm{CN}$. Pure g-CN has previously been presented as a metal-free visible light active disinfecting agent, suggesting that the obtained hydrogels may be toxic to microbial and mammalian cells. ${ }^{[37]}$ To test for possible toxicity of the hydrogel system, bacterial cultures (E. coli) were grown in the presence of hydrogel pieces under ambient light. In one experiment, the hydrogel was added to the culture medium right after synthesis without purification. Alternatively, the hydrogel was washed extensively with ultrapure water to remove non-crosslinked material. Bacterial growth curves from these two experiments were compared to a negative control where no hydrogel was added (Figure 2c). Upon addition of the non-purified hydrogel, insoluble aggregates appeared in the culture medium and the optical density at $600 \mathrm{~nm}\left(O D_{600}\right)$ increased in the first few minutes. We attribute this to g-CN leaching out of the hydrogel into the culture medium (Figure S7, Table S4, Supporting Information). Afterwards, no further increase in the $O D_{600}$ value was observed, suggesting that the non-crosslinked $\mathrm{g}-\mathrm{CN}$ is indeed toxic to the bacteria. In contrast, the washed hydrogel did not inhibit bacterial growth when compared to the control without hydrogel. This is a strong indication that the investigated hydrogels are nontoxic when non-crosslinked $\mathrm{g}-\mathrm{CN}$ is removed via extensive washing. The investigation of potential bio-related applications as well as mammalian cell compatibility will be studied in more detail in future projects.
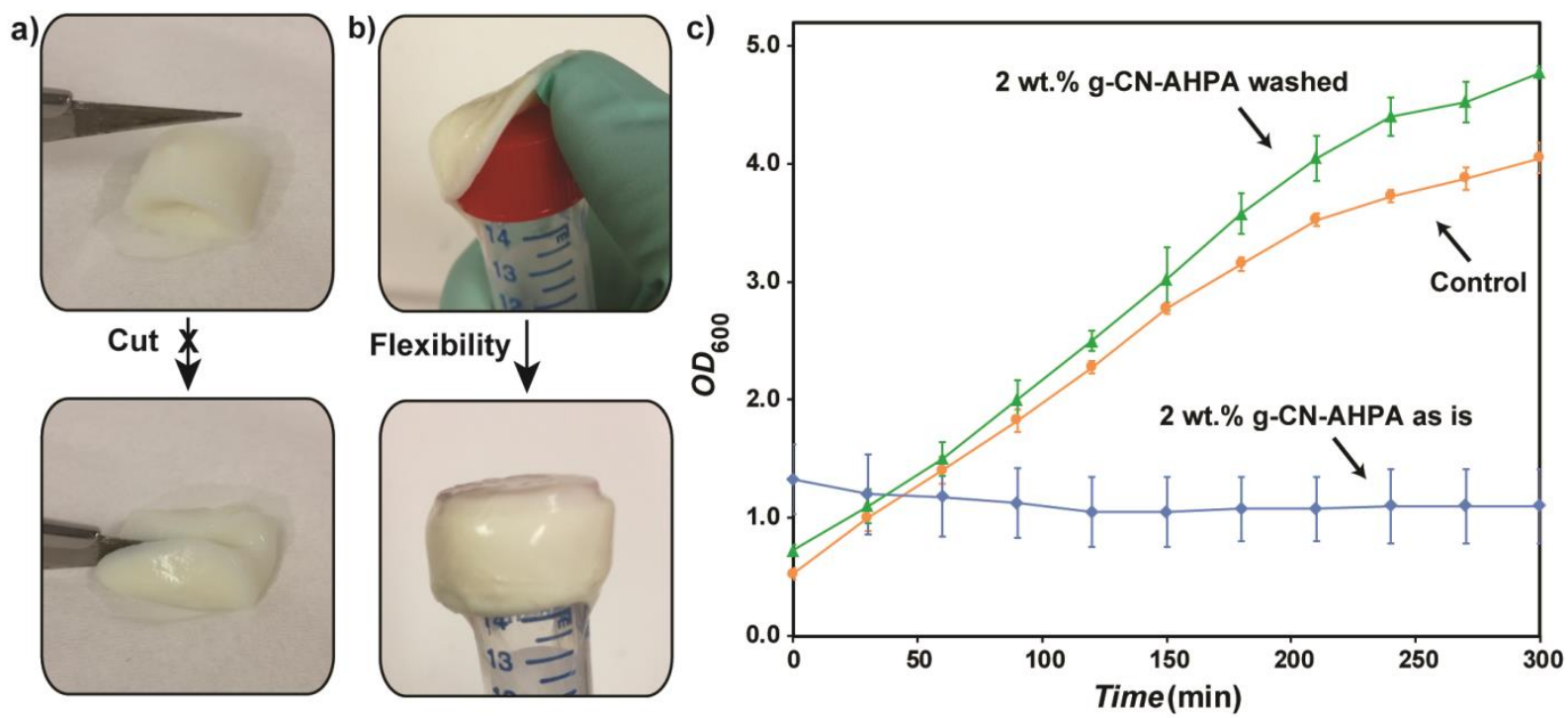

Figure 2. 2 wt.\% g-CN-AHPA hydrogel a) under compression with blade, b) hydrogel covering the poly(propylene) (PP) cap of a centrifuge tube, c) bacteria growth tests, showing 
an increase in the optical density at $600 \mathrm{~nm}\left(O D_{600}\right)$ in the presence of the washed hydrogel, whereas growth is inhibited for the non-purified hydrogels used directly after synthesis (blue data: 2 wt.\% g-CN-AHPA hydrogels without washing; green data: washed 2 wt.\% g-CNAHPA hydrogels; orange data: control samples) The data represents the mean and the standard deviation obtained from 4 independent experiments.

The presented hydrogel strongly adheres to tissue, e.g. to skin after gentle touch (Figure S8, Supporting Information). Skin-like feeling and bioadhesive properties may be of interest for biomedical applications, such as wound dressings and surgical sealants. ${ }^{[38,39]}$ The hydrogel does not only adhere to skin, but also to a variety of surfaces such as glass, metal, paper and poly(propylene) (PP) (Figure S9). During compression experiments, the force is applied at a gradual rate, giving the material the chance to absorb the stress with time. In a shock-type compression, stress is received non-gradually and immediately, which is a property that needs to be improved for hydrogels. ${ }^{[40]}$ To visualize shock absorption, the present hydrogel was hit with a $3 \mathrm{~kg}$ weight hammer (Video S6, Supporting Information), and no sign of fracture was observed.

Figure 3
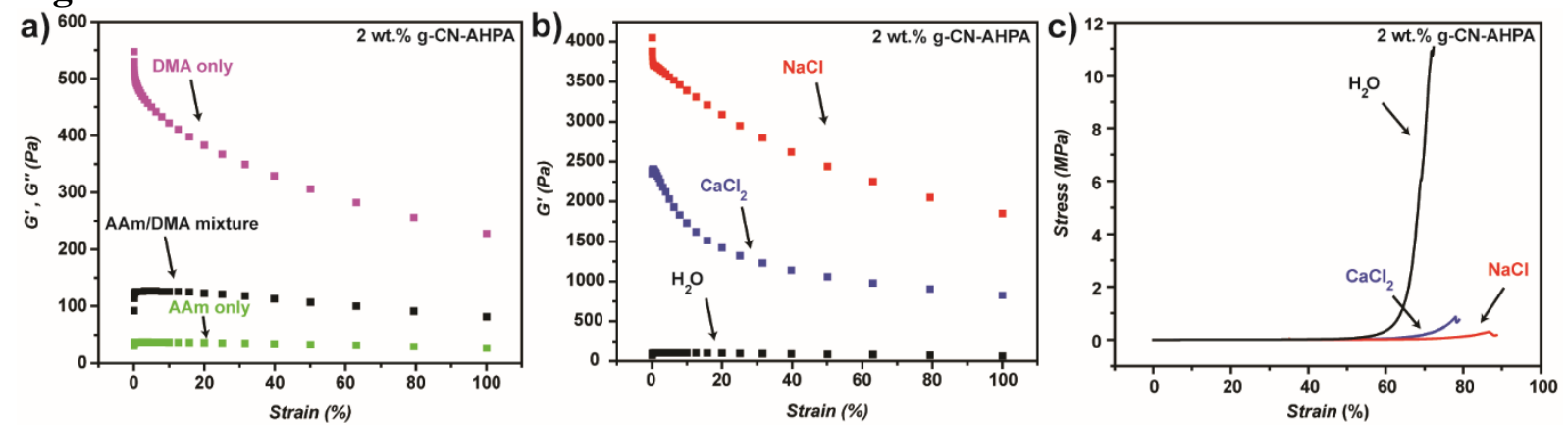

Figure 3. a) Rheology of 2 wt. $\%$ g-CN-AHPA hydrogel from different monomers (black squares: AAm/DMA mixture; violet squares: pure AAm; green squares: pure DMA), b) Rheology of fully swollen 2 wt.\% g-CN-AHPA hydrogel (black squares: in water; red squares: $\mathrm{NaCl}$ solution; blue squares: $\mathrm{CaCl}_{2}$ solution) and c) compression profiles of fully swollen 2 wt.\% g-CN-AHPA hydrogels (black line: in water; red line: $\mathrm{NaCl}$ solution; blue line: $\mathrm{CaCl}_{2}$ solution).

Swelling in water is an important property of hydrogels. Usually, addition of reinforcer (such as clay) decreases water uptake due to increased crosslinking density and hydrophobic interactions. ${ }^{[41]}$ Hence, it is a challenging task to improve mechanical and preserve swelling properties of hydrogels simultaneously. For the swelling properties, interestingly 2 wt. $\%$ g-CN-AHPA showed the highest swelling ratio with $2476 \%$, yet still showing a similar rheology profile and compressibility to the one right after gelation (Figure 3a). The preservation of mechanical properties in the presence of high amounts of water is a significant feature of the presented hydrogel material. 5 wt.\% g-CN-AHPA swells the least (254\%) due to a higher amount of reinforcer and increased crosslinking, and $0.35 \mathrm{wt}$ \% gCN-AHPA has a swelling ratio of $1750 \%$, which is still considerably high for hydrogel systems. Interestingly, swelling in acidic or basic media significantly affects the hydrogel structure. In the case of $2 \mathrm{wt} . \% \mathrm{~g}$-CN-AHPA hydrogel, swollen in $0.1 \mathrm{M} \mathrm{HCl}$ and $0.1 \mathrm{M}$ $\mathrm{NaOH}$ solutions, rheology of both hydrogels showed a significant decrease in $G^{\prime}$ and $G^{\prime}$ ' values, yet showing a similar strain dependency (Figure S10a-b, Supporting Information). The 
swelling ratio in basic media is almost 3 times higher than in acidic media (3855\% to $1310 \%$ ) (Figure S8c), which can be attributed to the incorporated sulfonic acid group.

To gain a deeper understanding of the toughening mechanism of $2 \mathrm{wt} \%$ g-CN-AHPA, hydrogel control reactions were performed. First of all, a comparison with hydrogels formed from a single monomer was investigated. 2 wt. $\%$ g-CN-AHPA hydrogel from AAm shows a soft rheology profile with good recovery $\left(G^{\prime} 30 \mathrm{~Pa}\right.$ at $0.1 \%$ strain). On the other hand, hydrogel from DMA is almost five times stronger than the 3:1 monomer mixture $(550 \mathrm{~Pa}$ at $0.1 \%$ strain), while a significant strain dependency and a yield stress of approximately $150 \mathrm{~Pa}$ is observed (Figure 3a/Figure S11, Supporting Information). The utilization of monomer mixtures increases the $G$ ' values of the DMA hydrogel compared to the AAm hydrogel (effect from DMA). In addition, a preserved recovery profile is obtained (effect from AAm). However, hydrogels from individual monomers break at gentle compression. Performed control experiments provide a hint for the necessity of using monomer mixtures with certain g-CN-AHPA ratio, which leads to a softening of the gel and a significant decrease in shear thinning behavior. Thus, the applied compression force can be effectively distributed over the whole network.

As shown in compressions tests, there was a non-linear increase in $G$ ' and $G$ "' values with increased g-CN-AHPA content, which may be due to amplified sheet-sheet interactions. Similar to the optimum monomer ratio, there is also an optimum amount of g-CN-AHPA incorporation to balance between stiffness of the hydrogel and elasticity. Conclusively the negative charge on the g-CN surface plays an important role for reinforcement. ${ }^{[27]}$ Thus, swelling of 2 wt.\% g-CN-AHPA hydrogels in salt solutions $\left(0.2 \mathrm{M}\right.$ in $\mathrm{NaCl}$ or $\left.\mathrm{CaCl}_{2}\right)$ was performed. Compared with the water swollen hydrogel, swelling in salt media significantly increases $G$ ' and $G$ ' 'values by gaining highly shear thinning behavior, which may be due to possible cluster formation, originating from cation of salt-anion on g-CN-AHPA surface interactions (Figure S12, Supporting Information). ${ }^{[42]}$ Another reason for the increased $G$ ' values are increased sheet-sheet interactions, which is in-line with zeta potential measurements of g-CN-AHPA in salt solution (Table S5). However, hydrogels swollen in salt solution are easy-to-break upon compression at forces around $100 \mathrm{~N}$ (Figure 3c). Also, the elastic moduli values decrease in salt swollen hydrogels, compared to the water swollen reference (1.15 MPa for fully water swollen, $0.64 \mathrm{MPa}$ for $\mathrm{NaCl}$ swollen and $1.47 \mathrm{MPa}$ for $\mathrm{CaCl}_{2}$ swollen hydrogel). Zeta potential measurements of aqueous dispersions of g-CNAHPA were monitored at different $\mathrm{pH}$ and with different salts (Table S5, Supporting Information). Indeed, changing $\mathrm{pH}$ or introducing salt affects surface charges of $\mathrm{g}-\mathrm{CN}$, which ultimately affects the strength of the hydrogel network. For example, after salt addition the zeta potential of pure g-CN-AHPA increases, which leads to less repulsion of the g-CN sheets in the hydrogel and thus less compressibility. Preserving negative charge on g-CN surface inside the hydrogel possibly plays the most important role for the extreme compressibility due to strong repulsion of negatively charged sheets upon stress. Decreasing g-CN-AHPA content increases polymer network density per sheet, which may hinder the repulsion of sheets upon stress. Oppositely, increasing g-CN-AHPA content decreases the polymer network density per sheet, and $\pi-\pi$ interactions of $\mathrm{g}-\mathrm{CN}$ sheets become more pronounced and provide toughness (increased $G$ 'values), yet still fail to improve compression upon stress.

Overall, we report novel reinforced soft hydrogel materials. The hydrogels feature outstanding mechanical properties such as compressibility, cut and shock resistance as well as tissue adhesive properties with moderate flexibility. Sulfonic acid modified g-CN is utilized as photoinitiator and covalent reinforcer under visible light for one pot hydrogel synthesis. Different concentrations of g-CN-AHPA can be introduced while retaining high amounts of water (up to $99 \mathrm{wt} . \%$ ). A weight content of 2 and $3.5 \mathrm{wt} . \% \mathrm{~g}-\mathrm{CN}$-AHPA leads to hydrogels that possess excellent compressibility and withstand loads up to $12 \mathrm{MPa}(800 \mathrm{~N})$ and show elastic behavior without loss for 50 cycles at $2 \mathrm{MPa}(100 \mathrm{~N})$ loading. The presented hydrogels 
show similar resistance when the stress is received non-uniformly. We believe that the presented hydrogel can be of great interest in bioapplications such as soft tissue engineering, wound healing, joint replacement and surgical sealant. Due to the skin-like soft feeling, the gCN-AHPA hydrogel might replace PDMS in wearable electronics in the near future.

\section{Supporting Information}

Supporting Information is available via

https://onlinelibrary.wiley.com/doi/full/10.1002/marc.201800712

\section{Acknowledgements}

The authors thank Max Planck Society for financial support.

\section{References}

[1] E. Caló and V. V. Khutoryanskiy, Eur. Polym. J., 2015, 65, 252-267.

[2] X. Dai, Y. Zhang, L. Gao, T. Bai, W. Wang, Y. Cui and W. Liu, Adv. Mater., 2015, 27, 3566-3571.

[3] J. P. Gong, Soft Matter, 2010, 6, 2583.

[4] L. Wang, G. Shan and P. Pan, Soft Matter, 2014, 10, 3850-3856.

[5] L. Si, X. Zheng, J. Nie, R. Yin, Y. Hua and X. Zhu, Chem. Commun. 2016, 52, 83658368 .

[6] T. Huang, H. G. Xu, K. X. Jiao, L. P. Zhu, H. R. Brown and H. L. Wang, Adv. Mater., 2007, 19, 1622-1626.

[7] S. K. Mujumdar and R. A. Siegel, J. Polym. Sci., Part A: Polym. Chem., 2008, 46, 6630-6640.

[8] L. W. Xia, R. Xie, X. J. Ju, W. Wang, Q. Chen and L. Y. Chu, Nat. Commun., 2013, 4, 2226.

[9] Z. Wang, Y. Ren, Y. Zhu, L. Hao, Y. Chen, G. An, H. Wu, X. Shi and C. Mao, Angew. Chem., Int. Ed., 2018, 57, 9008-9012.

[10] T. Li, B. Kumru, N. Al Nakeeb, J. Willersinn and B. V. K. J. Schmidt, Polymers, 2018, 10,576 .

[11] M. Liu, Y. Ishida, Y. Ebina, T. Sasaki, T. Hikima, M. Takata and T. Aida, Nature, 2015, 517, 68-72.

[12] Q. Wang, J. L. Mynar, M. Yoshida, E. Lee, M. Lee, K. Okuro, K. Kinbara and T. Aida, Nature, 2010, 463, 339-343.

[13] L. Liu, L. Li, Y. Qing, N. Yan, Y. Wu, X. Li and C. Tian, Polym. Chem., 2016, 7, 7142-7151.

[14] A. K. Gaharwar, N. A. Peppas and A. Khademhosseini, Biotechnol. Bioeng., 2014, $111,441-453$.

[15] M. Jing, Y. Fu, X. Fei, J. Tian, H. Zhi, H. Zhang, L. Xu, X. Wang and Y. Wang, Polym. Chem., 2017, 8, 3553-3559.

[16] T. Bai, P. Zhang, Y. Han, Y. Liu, W. Liu, X. Zhao and W. Lu, Soft Matter, 2011, 7, 2825.

[17] A. Wang, C. Wang, L. Fu, W. Wong-Ng and Y. Lan, Nano-Micro Lett., 2017, 9. 
[18] Q. Zheng, D. P. Durkin, J. E. Elenewski, Y. Sun, N. A. Banek, L. Hua, H. Chen, M. J. Wagner, W. Zhang and D. Shuai, Environ. Sci. Technol., 2016, 50, 12938-12948.

[19] Q. Han, B. Wang, Y. Zhao, C. Hu and L. Qu, Angew. Chem., Int. Ed., 2015, 54, 11433-11437.

[20] J. Mao, T. Peng, X. Zhang, K. Li, L. Ye and L. Zan, Catal. Sci. Technol., 2013, 3, 1253.

[21] J. Xu and M. Antonietti, J. Am. Chem. Soc., 2017, 139, 6026-6029.

[22] F. Su, S. C. Mathew, L. Mohlmann, M. Antonietti, X. Wang and S. Blechert, Angew. Chem., Int. Ed., 2011, 50, 657-660.

[23] B. Kurpil, B. Kumru, T. Heil, M. Antonietti and A. Savateev, Green Chem., 2018, 20, 838-842.

[24] Y. Zhang, Z. Zhou, Y. Shen, Q. Zhou, J. Wang, A. Liu, S. Liu and Y. Zhang, ACS Nano, 2016, 10, 9036-9043.

[25] S. Dadashi-Silab, M. A. Tasdelen, B. Kiskan, X. Wang, M. Antonietti and Y. Yagci, Macromol. Chem. Phys., 2014, 215, 675-681.

[26] Q. Fu, Q. Ruan, T. G. McKenzie, A. Reyhani, J. Tang and G. G. Qiao, Macromolecules, 2017, 50, 7509-7516.

[27] B. Kumru, M. Shalom, M. Antonietti and B. V. K. J. Schmidt, Macromolecules, 2017, 50, 1862-1869.

[28] J. W. Ko, W. S. Choi, J. Kim, S. K. Kuk, S. H. Lee and C. B. Park, Biomacromolecules, 2017, 18, 3551-3556.

[29] J. Sun, B. V. K. J. Schmidt, X. Wang and M. Shalom, ACS Appl. Mater. Interfaces, 2017, 9, 2029-2034.

[30] J. Liu, T. An, Z. Chen, Z. Wang, H. Zhou, T. Fan, D. Zhang and M. Antonietti, J. Mater. Chem. A, 2017, 5, 8933-8938.

[31] B. Kumru, V. Molinari, M. Shalom, M. Antonietti and B. V. K. J. Schmidt, Soft Matter, 2018, 14, 2655-2664.

[32] B. Kumru, M. Antonietti and B. V. K. J. Schmidt, Langmuir, 2017, 33, 9897-9906.

[33] M. Shalom, S. Inal, C. Fettkenhauer, D. Neher and M. Antonietti, J. Am. Chem. Soc., 2013, 135, 7118-7121.

[34] C. Deng, Y. Cui, T. Zhao, M. Tan, H. Huang and M. Guo, RSC Adv., 2014, 4, 2409524102.

[35] R. A. Brand, Iowa Orthop. J., 2005, 25, 82-94.

[36] A. Thambyah, J. C. Goh and S. D. De, Med. Eng. Phys., 2005, 27, 329-335.

[37] J. Huang, W. Ho and X. Wang, Chem. Comm., 2014, 50, 4338-4340.

[38] J. Li and D. J. Mooney, Nat. Rev. Mater., 2016, 1.

[39] C. W. Peak, J. J. Wilker and G. Schmidt, Colloid Polym. Sci., 2013, 291, 2031-2047.

[40] M. A. Haque, T. Kurokawa and J. P. Gong, Polymer, 2012, 53, 1805-1822.

[41] W. Huang, J. Shen, N. Li and M. Ye, Polym. Eng. Sci., 2015, 55, 1361-1366.

[42] C. Papadakis and C. Tsitsilianis, Gels, 2017, 3, 3. 


\section{Supporting Information}

for Macromol. Rapid Commun., DOI: 10.1002/marc.201800712

Extremely Compressible Hydrogel via Incorporation of Modified Graphitic Carbon Nitride

Baris Kumru, Valerio Molinari, Reinhild Dünnebacke, Kerstin G. Blank,* and Bernhard V. K. J. Schmidt* 


\section{Extremely Compressible Skin-Like Hydrogel via Incorporation of Modified Graphitic Carbon Nitride}

Baris Kumru, Valerio Molinari, Reinhild Dünnebacke, Kerstin G. Blank, * Bernhard V. K. J. Schmidt* Max-Planck-Institute of Colloids and Interfaces; Department of Colloid Chemistry, Am Mühlenberg 1, 14476 Potsdam, Germany

Max-Planck-Institute of Colloids and Interfaces; Mechano(bio)chemistry, Am Mühlenberg 1, 14476 Potsdam, Germany

Email: bernhard.schmidt@mpikg.mpg.de; kerstin.blank@mpikg.mpg.de

Materials: All materials were used as purchased unless noted otherwise. 3-Allyloxy-2hydroxy-1-propanesulfonic acid sodium salt solution (40\% w/w, AHPA, Sigma Aldrich), acrylamide (AAm, 98\% GC grade, Sigma Aldrich), $\mathrm{Al}_{2} \mathrm{O}_{3}$ basic (Sigma Aldrich), calcium chloride (97\%, Alfa Aesar), cyanuric acid (98\%, Sigma Aldrich), E. coli Turbo bacteria (New England Biolabs), hydrochloric acid solution (0.1 M, Sigma-Aldrich), melamine (99\%, Sigma Aldrich), N,N-methylenebisacrylamide (MBA, 99\%, Sigma Aldrich), sodium hydroxide solution (0.1 N, Sigma-Aldrich), sodium chloride (99\%, Sigma Aldrich). $N, N$ Dimethylacrylamide (DMA, 99\%, TCI) was passed through basic alumina column prior to use. Visible light irradiation was performed via two 50 W LED chips (Foxpic High Power 50 W LED Chip Bulb Light DIY White 3800LM $6500 \mathrm{~K}$ ) connected to a self-made circuit and cooling system.

Characterizations: Zeta potential measurement of colloidal suspension of g-CN and g-CNAHPA was performed with a Zetasizer Nano ZS90 from Malvern. X-ray diffraction (XRD) patterns were obtained using Bruker D8 Advance X-ray diffractometer via $\mathrm{Cu}-\mathrm{K}_{\alpha}$ radiation. Scanning electron microscopy (SEM) was performed using JSM-7500F (JEOL) equipped with an Oxford Instruments X-MAX $80 \mathrm{~mm}^{2}$ detector for the elemental mapping of g-CN and g-CN-AHPA samples. The structures of g-CN and g-CN-AHPA were also examined by TEM using an EM 912 Omega microscope at $120 \mathrm{kV}$. Fourier transform infrared (FT-IR) spectra were taken on Nicolet iS 5 FT-IR spectrometer. Solid state ultraviolet-visible (UV-Vis) spectroscopy was recorded via a Cary 500 Scan spectrophotometer equipped with an integrating sphere. Elemental analysis of g-CN and g-CN-AHPA was recorded via a Vario Micro device. Swelling and rheological analysis of hydrogel samples was performed as follows. To obtain the swelling ratios of hydrogel samples manually $100 \mathrm{mg}\left(W_{\mathrm{d}}\right)$ freeze dried hydrogel sample were put into a flask which contains $10 \mathrm{~mL}$ distilled water and left to stand 
for 36 hours. Swollen hydrogels are weighed $\left(W_{\mathrm{s}}\right)$ and swelling ratio is calculated by using the formula:

$$
\text { Swelling ratio }=\frac{W s-W d}{W d} \times 100 \%
$$

For rheological investigations, purified and swollen hydrogels were cut into small disc shapes and investigated with an Anton Paar MCR 301 rheometer, equipped with a cone plate 12 (CP12). Measurements were performed at constant angular frequency $\left(10 \mathrm{rad} \mathrm{s}^{-1}\right)$ with strain range from $0.1-100 \%$ with 31 measuring points and $0.02 \mathrm{~mm}$ gap. Frequency dependent measurements were performed at constant strain $(0.1 \%)$ with changing frequency in the range of 1-100 $\mathrm{rad} \mathrm{s}^{-1}$. Compression measurements were measured on a Zwick mechanical tester zwickiLine Z2.5 equipped with a loadcell of $1 \mathrm{kN}$. Measurements were recorded after a preload of $0.1 \mathrm{~N}$ (speed pre-load $40 \mathrm{~mm} \mathrm{~s}^{-1}$ ) and the test was performed at $0.05 \mathrm{~mm} \mathrm{~s}^{-1}$. The cycling tests were performed until $100 \mathrm{~N}$ (or $800 \mathrm{~N}$ ) of maximum force and recovered until 1 $\mathrm{mm}$ of strain (on specimens of $10 \mathrm{~mm}$ in height) before the stress was removed to restart the cycle (50 times). Elastic modulus was manually calculated at $10 \%$ of strain before break for every specimen. As the compression profiles of 2 and 3.5 wt.\% g-CN-AHPA hydrogels show dramatic increase instead of linear growth, elastic modulus was calculated at the increase point in addition. Compression strength of 2 wt. \% g-CN-AHPA hydrogel was calculated at the maximum point on the stress-strain curve. All the compression measurements were recorded using the software TestXpert II V3.71.

\section{Experimental}

Synthesis of Graphitic Carbon Nitride (g-CN): g-CN was prepared from cyanuric acidmelamine $(\mathrm{CM})$ complex as reported in literature. ${ }^{1}$ In a typical process, $1.0 \mathrm{~g}$ of cyanuric acid and $1.0 \mathrm{~g}$ of melamine were mixed with $40 \mathrm{~mL}$ distilled water and shaked overnight. After centrifugation at $5000 \mathrm{rpm}$ for 10 minutes, a precipitate was dried at $60{ }^{\circ} \mathrm{C}$ under vacuum overnight. The dried product was transferred into a capped crucible and put into $\mathrm{N}_{2}$ protected oven at $550{ }^{\circ} \mathrm{C}$ for 4 hours, with a heating rate of $2.3{ }^{\circ} \mathrm{C} / \mathrm{min}$.

Synthesis of g-CN-AHPA: g-CN was hydrophilically modified using AHPA solution as previously reported by our group. ${ }^{2}$ In a typical process, $200 \mathrm{mg}$ g-CN was mixed with $2 \mathrm{~g}$ AHPA solution and $2 \mathrm{~g}$ distilled water and sonicated for 5 minutes. After nitrogen flow through solution for 5 minutes, the mixture was put between 2 LEDs on visible light range 
and stirred for 12 hours. It was then filtered, washed with distilled water ( 3 times $-50 \mathrm{~mL}$ distilled water each) for removal of unreacted portions and finally washed with $20 \mathrm{~mL}$ acetone. It was dried overnight in vacuum oven at $50{ }^{\circ} \mathrm{C}$.

Synthesis of Hydrogel With 0.35\% g-CN-AHPA Content: $35 \mathrm{mg}$ g-CN-AHPA was mixed with $9.9 \mathrm{~g}$ distilled water in a plastic vessel and sonicated for 2 minutes for homogenous dispersion of g-CN-AHPA particles. $45 \mathrm{mg}$ AAm, $15 \mathrm{mg}$ DMA and $5 \mathrm{mg}$ MBA was added to the mixture, sealed and put between 2 LED visible light sources until complete gelation (approx. 20 hours).

Synthesis of Hydrogel With 1\% g-CN-AHPA Content: $100 \mathrm{mg}$ g-CN-AHPA was mixed with $9.1 \mathrm{~g}$ distilled water in a plastic vessel and sonicated for 5 minutes for homogenous dispersion of g-CN-AHPA particles. $600 \mathrm{mg}$ AAm, $200 \mathrm{mg}$ DMA and $3 \mathrm{mg}$ MBA was added to the mixture, sealed and put between 2 LED visible light sources until complete gelation (approx. 10 hours).

Synthesis of Hydrogel With 2\% g-CN-AHPA Content: $200 \mathrm{mg}$ g-CN-AHPA was mixed with $9 \mathrm{~g}$ distilled water in a plastic vessel and sonicated for 5 minutes for homogenous dispersion of g-CN-AHPA particles. $600 \mathrm{mg}$ AAm, $200 \mathrm{mg}$ DMA and $3 \mathrm{mg}$ MBA was added to the mixture, sealed and put between 2 LED visible light sources until complete gelation (approx. 3 hours).

Synthesis of Hydrogel With 3.5\% g-CN-AHPA Content: 350 mg g-CN-AHPA was mixed with $8.85 \mathrm{~g}$ distilled water in a plastic vessel and sonicated for 5 minutes for homogenous dispersion of g-CN-AHPA particles. $600 \mathrm{mg}$ AAm, $200 \mathrm{mg}$ DMA and $3 \mathrm{mg}$ MBA was added to the mixture, sealed and put between 2 LED visible light sources until complete gelation (approx. 4 hours).

Synthesis of Hydrogel With 5\% g-CN-AHPA Content: $500 \mathrm{mg}$ g-CN-AHPA was mixed with $8.7 \mathrm{~g}$ distilled water in a plastic vessel and sonicated for 10 minutes for homogenous dispersion of g-CN-AHPA particles. $600 \mathrm{mg}$ AAm, $200 \mathrm{mg}$ DMA and $3 \mathrm{mg}$ MBA was added to the mixture, sealed and put between 2 LED visible light sources until complete gelation (approx. 2 hours).

Synthesis of AAm Hydrogel With 2\% g-CN-AHPA Content: $200 \mathrm{mg}$ g-CN-AHPA was mixed with $9 \mathrm{~g}$ distilled water in a plastic vessel and sonicated for 5 minutes for homogenous 
dispersion of g-CN-AHPA particles. $800 \mathrm{mg}$ AAm and $3 \mathrm{mg}$ MBA was added to the mixture, sealed and put between 2 LED visible light sources until complete gelation (approx. 7 hours).

Synthesis of DMA Hydrogel With $2 \%$ g-CN-AHPA Content: $200 \mathrm{mg}$ g-CN-AHPA was mixed with $9 \mathrm{~g}$ distilled water in a plastic vessel and sonicated for 5 minutes for homogenous dispersion of g-CN-AHPA particles. $800 \mathrm{mg}$ DMA and $3 \mathrm{mg}$ MBA was added to the mixture, sealed and put between 2 LED visible light sources until complete gelation (approx. 3 hours).

Investigation of Toxicity in 2\% g-CN-AHPA Hydrogel: To test the biocompatibility of the hydrogel, E. coli cultures were grown in the presence and absence of the hydrogel. The hydrogel was either used directly as obtained from the synthesis or washed extensively with ultrapure water to remove non-crosslinked material (Figure S7; Table S4). Bacterial growth was monitored over time, measuring the optical density at $600 \mathrm{~nm}\left(O D_{600}\right)$. In detail, a preculture of fast-growing E. coli Turbo bacteria was grown over night $\left(37^{\circ} \mathrm{C}, 250 \mathrm{rpm}\right)$ in lysogeny broth (LB; $10 \mathrm{~g} / 1$ tryptone, $5 \mathrm{~g} / 1$ yeast extract, $10 \mathrm{~g} / 1 \mathrm{NaCl}$ ). Using this pre-culture, 3 main cultures were prepared with $O D_{600}=0.1(10 \mathrm{ml} \mathrm{LB})$. The cultures were grown under ambient light at $37{ }^{\circ} \mathrm{C}$ and $250 \mathrm{rpm}$ until they reached $O D_{600}=0.5-0.6$. At this point $\left(\mathrm{t}_{0}=\right.$ $0 \mathrm{~min}), 1 \mathrm{~g}$ of 'as is' or washed hydrogel was added to the cultures. In the case of the washed hydrogel, the weight was determined before washing. As a control, the same amount of sterile, ultrapure water was added instead of hydrogel. In the next 5.5 hours, samples were taken in $30 \mathrm{~min}$ intervals for measuring $O D_{600}$. The obtained $O D_{600}$ values were used for plotting growth curves. Each condition was measured 4 times, using independent pre-cultures.

Table S1. Properties of g-CN and g-CN-AHPA.

\begin{tabular}{|l|l|l|l|}
\hline & $\begin{array}{l}\text { Surface Zeta } \\
\text { Potential }(\mathbf{m V})^{\mathbf{a}}\end{array}$ & C:N ratio $^{\mathbf{b}}$ & S content (wt. \%) \\
\hline g-CN & -35.5 & 0.6083 & 0.816 \\
\hline g-CN-AHPA & -50.8 & 1.0783 & 6.143 \\
\hline
\end{tabular}

${ }^{\mathrm{a}}$ Obtained by water dispersion of particles, ${ }^{\mathrm{b}}$ obtained by elemental analysis 
a)

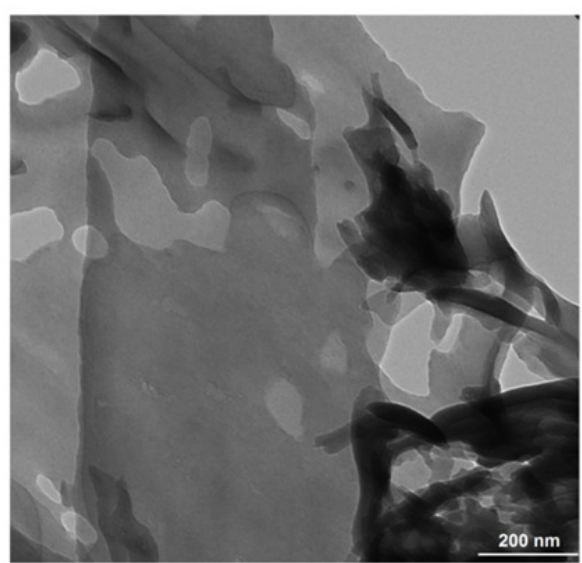

b)

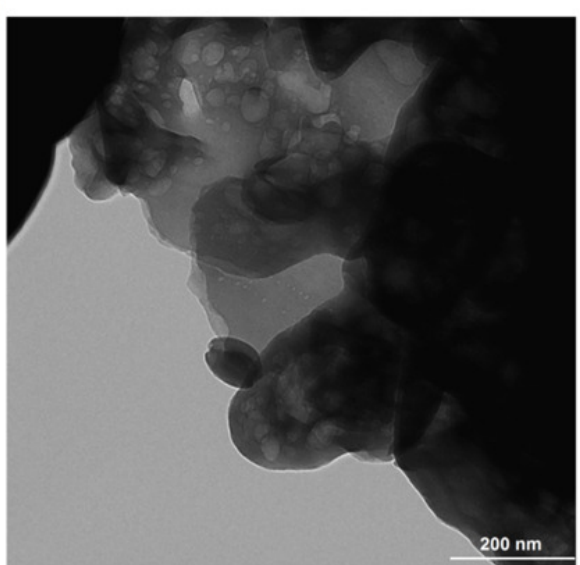

Figure S1. TEM images of a) g-CN and b) g-CN-AHPA.
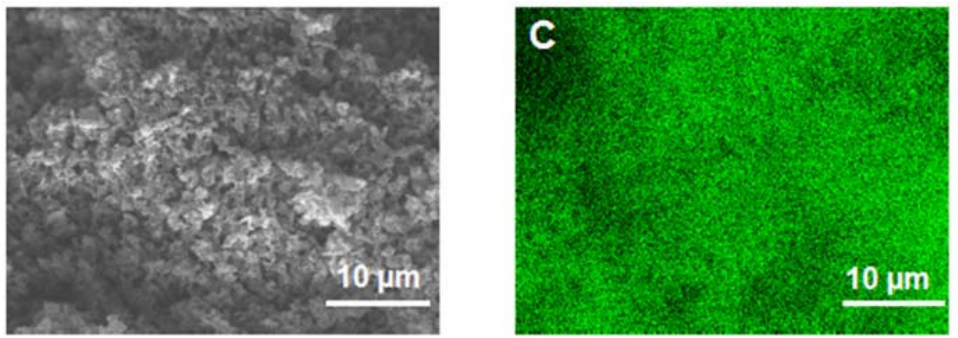

s

$\mathrm{Na}$

$10 \mu \mathrm{m}$

$10 \mu \mathrm{m}$

Figure S2. Elemental mapping of g-CN-AHPA via SEM. 
a)

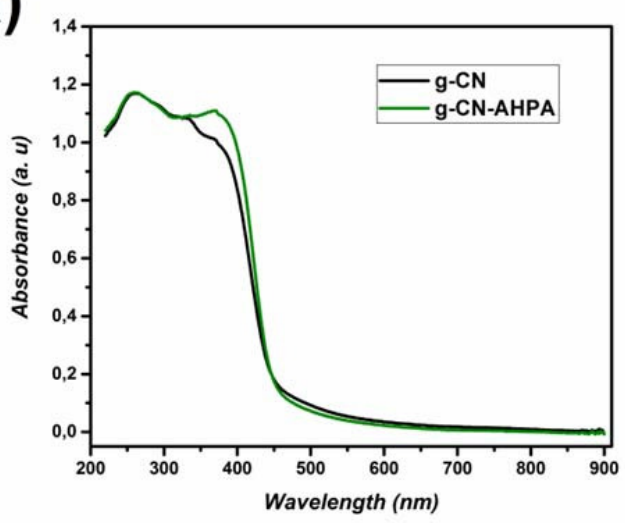

b)

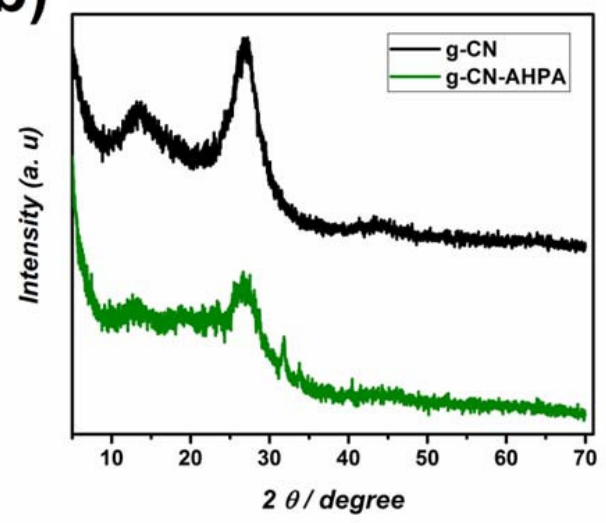

c)

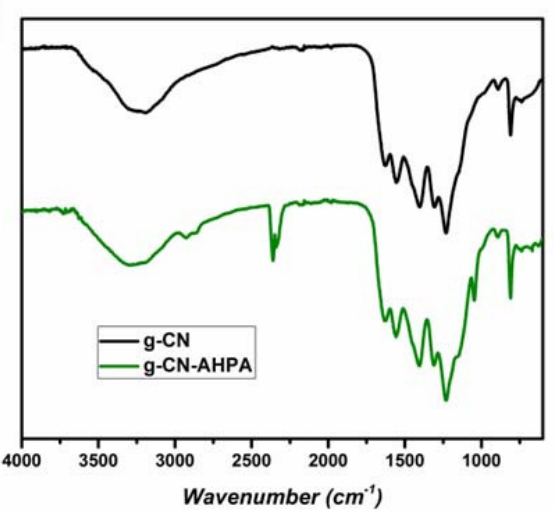

Figure S3. Solid state analysis results of g-CN and g-CN-AHPA; a) UV-Vis, b) XRD and c) FT-IR. 
a)

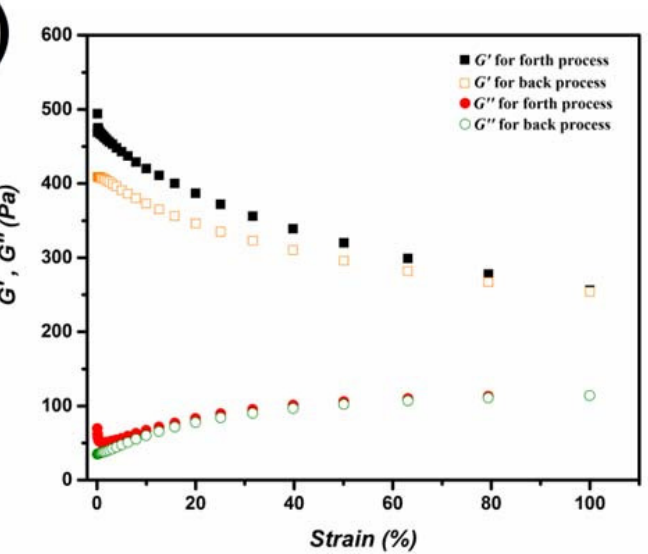

b)

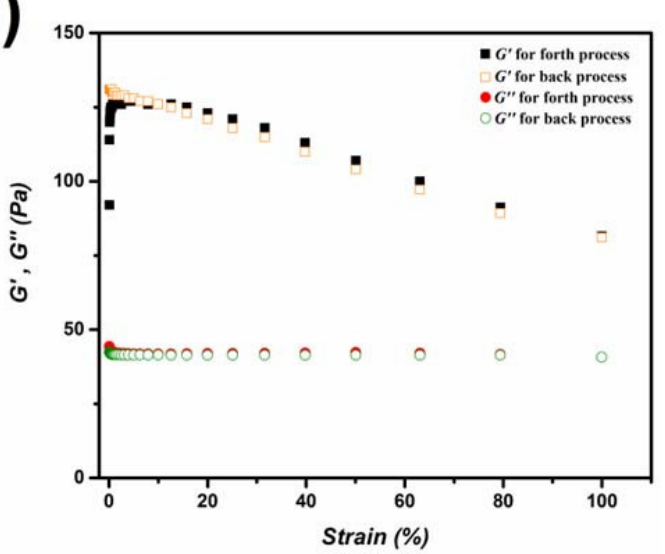

c)

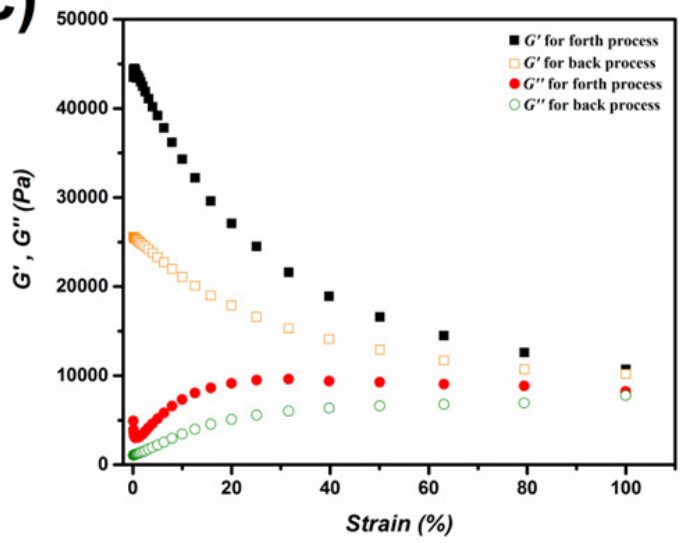

d)

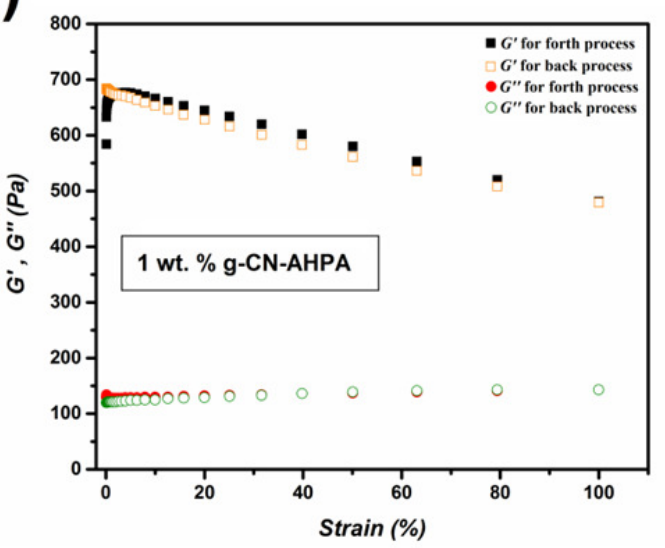

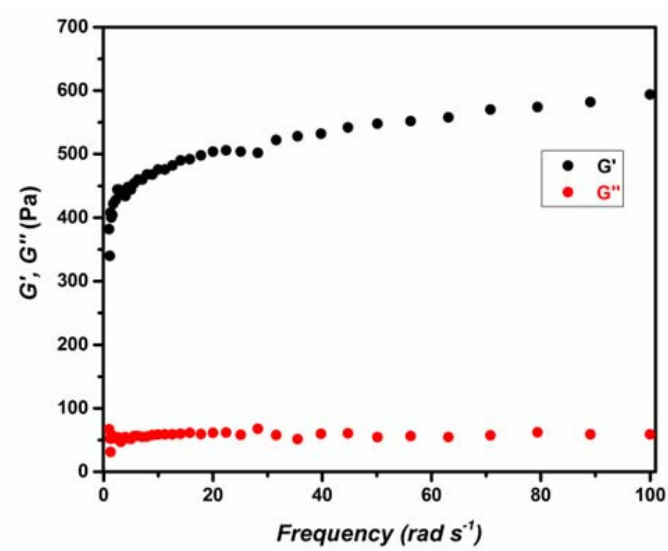
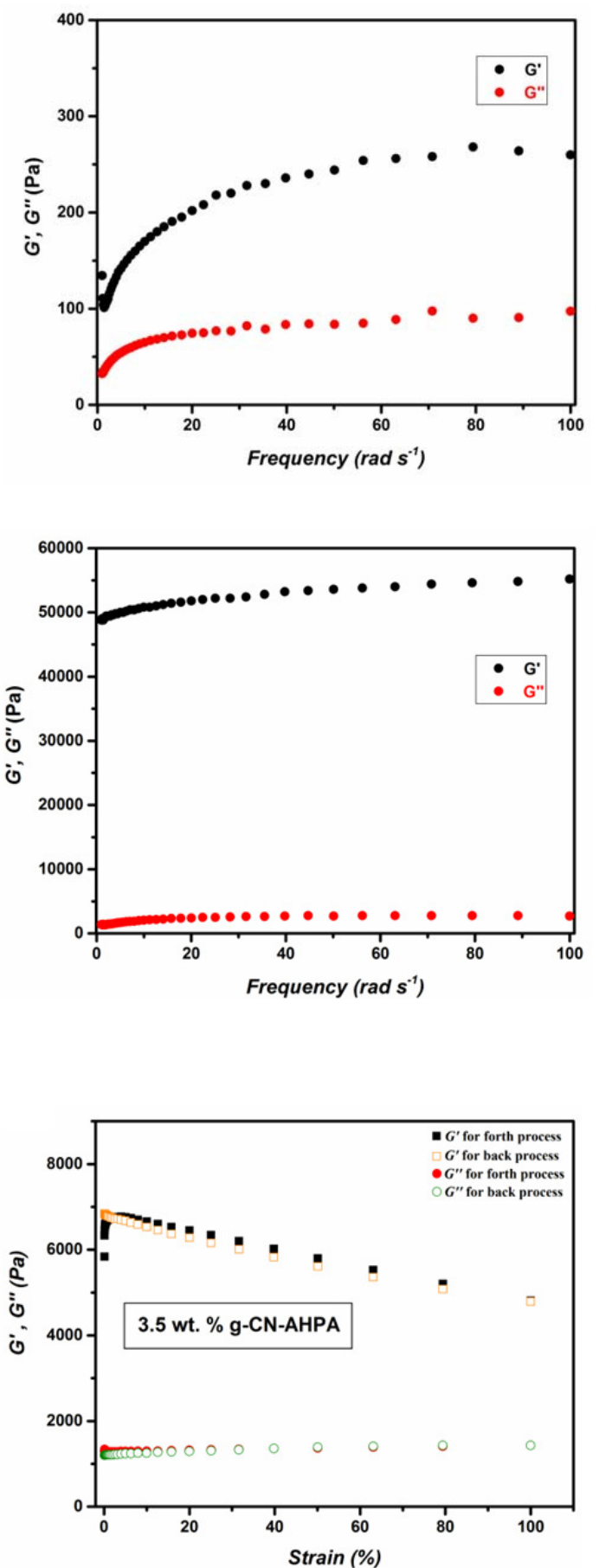
Figure S4. Comparison of storage and loss modulus values of a) 0.35 wt. $\%$ g-CN-AHPA, b) 2 wt.\% g-CN-AHPA and c) 5 wt.\% g-CN-AHPA hydrogels against strain (left, $G^{\prime}$ values are black and orange squares and $G$ ' values are red and green circles, back (open) and forth (filled) process) and frequency (right), d)storage and loss modulus values of $1 \mathrm{wt} . \% \mathrm{~g}-\mathrm{CN}$ AHPA (left) and 3.5 wt.\% g-CN-AHPA hydrogels

a)

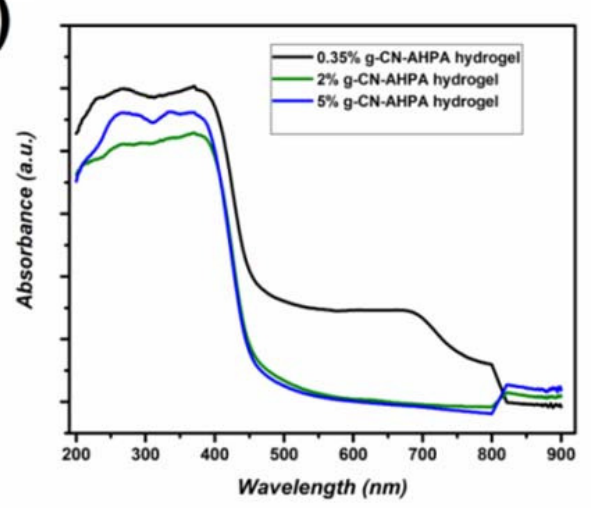

c)

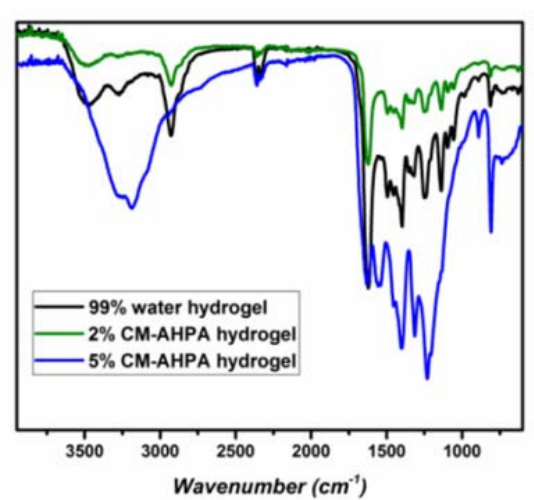

b)

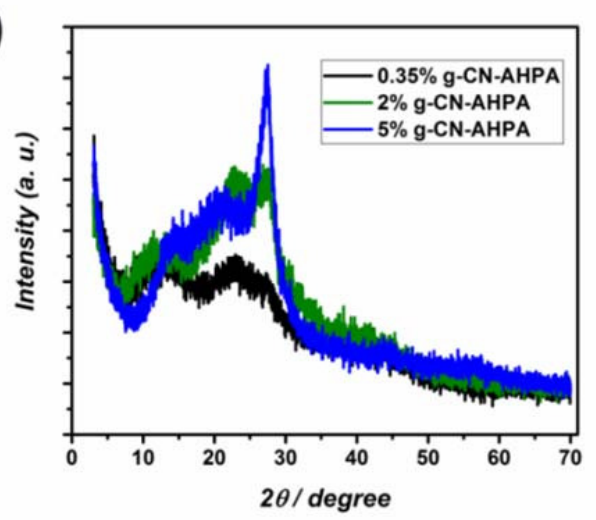

d)

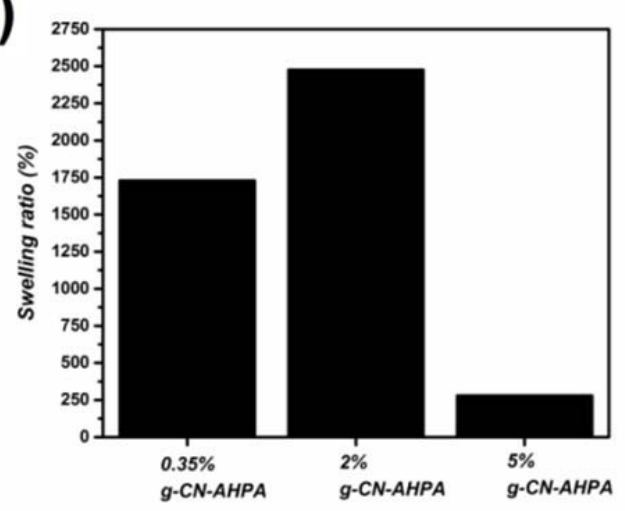

Figure S5. A) UV-Vis spectra, b) XRD profiles, c) FT-IR results and d) swelling ratios of gCN-AHPA derived hydrogels. 

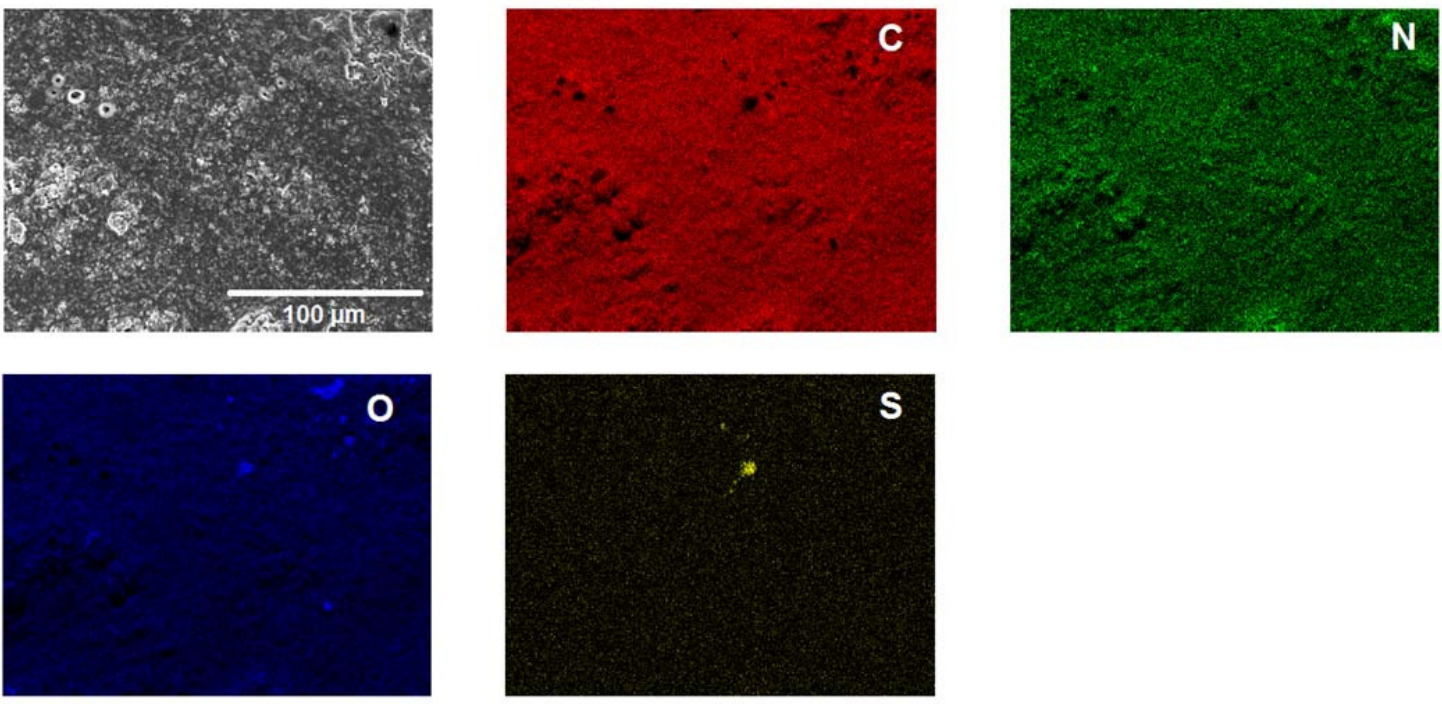

Figure S6. SEM image of freeze dried 2 wt.\% g-CN-AHPA hydrogel and relative EDX mapping results.

Table S2. Elastic moduli values of hydrogels calculated right before the break or at the slope where increase starts.

\begin{tabular}{|c|c|c|c|}
\hline $\begin{array}{c}\text { g-CN-AHPA content } \\
\text { of hydrogel (wt. \%) }\end{array}$ & $\begin{array}{c}\text { Strain at break } \\
(\boldsymbol{\%})\end{array}$ & $\begin{array}{c}\text { Elastic moduli } \\
(\mathbf{M P a})\end{array}$ & $\begin{array}{c}\text { Maximum stress } \\
(\mathbf{M P a})\end{array}$ \\
\hline 0.35 & 73.8 & 1.7 & 0.13 \\
\hline 1 & 68.7 & 6.7 & 0.42 \\
\hline 2 & - & 64.1 & 3.23 \\
\hline 3.5 & - & 68.7 & 11.92 \\
\hline 5 & 57.5 & 0.47 & 0.04 \\
\hline
\end{tabular}


Table S3. Comparison of compression strength and water content values of present hydrogel with literature values (for non-deformed systems).

\begin{tabular}{|l|l|l|}
\hline Reinforcement type & Water Content (\%) & $\begin{array}{l}\text { Compressive } \\
\text { Strength (MPa) }\end{array}$ \\
\hline Covalent (present hydrogel) & 90 & 18 \\
\hline Supramolecular & 30 & 34 \\
\hline Microsphere composite $^{4}$ & 46 & 10.2 \\
\hline Interpenetrating network $^{5}$ & 50.2 & 91.8 \\
\hline Dipole-dipole $^{6}$ & 60.4 & 13.9 \\
\hline Metal coordination $^{7}$ & 41 & 16 \\
\hline
\end{tabular}

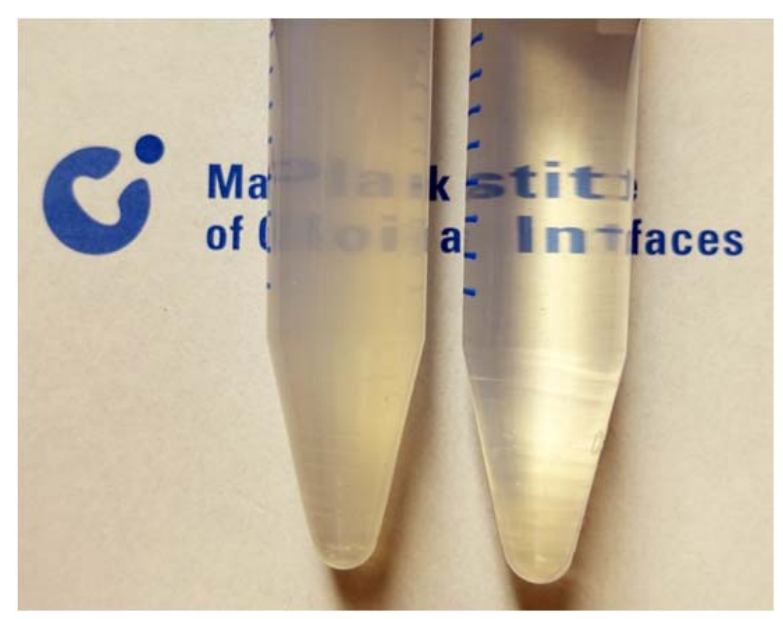

Figure S7. Supernatant $(10 \mathrm{ml})$ after washing the $2 \% \mathrm{~g}$-CN-AHPA hydrogel in ultrapure water for 2 hours (left; first washing step in Table S4). The sample on the right shows ultrapure water as a comparison. 
Table S4. Leakage observed when washing the $2 \%$ g-CN-AHPA hydrogel in ultrapure water. The amount of leaked material was determined as the optical density at $600 \mathrm{~nm}\left(\mathrm{OD}_{600}\right)$.

\begin{tabular}{|l|l|l|l|l|}
\hline Hydrogel & Mass $(\mathbf{g})$ & $\begin{array}{l}\text { Wash 1 } \\
(\mathbf{2} \mathbf{~ h}, \mathbf{1 0} \mathbf{~ m l})\end{array}$ & $\begin{array}{l}\text { Wash 2 } \\
(\mathbf{3} \mathbf{~ h}, \mathbf{2 0} \mathbf{~ m l})\end{array}$ & $\begin{array}{l}\text { Wash 3 } \\
(\mathbf{1 2} \mathbf{~ h}, \mathbf{3 5} \mathbf{~ m l})\end{array}$ \\
\hline 1 & 1.021 & 0.55 & 0.01 & 0.01 \\
\hline 2 & 1.024 & 0.80 & 0.02 & 0.01 \\
\hline 3 & 1.045 & 0.22 & 0.01 & 0.01 \\
\hline 4 & 1.014 & 0.32 & 0.01 & 0.00 \\
\hline
\end{tabular}
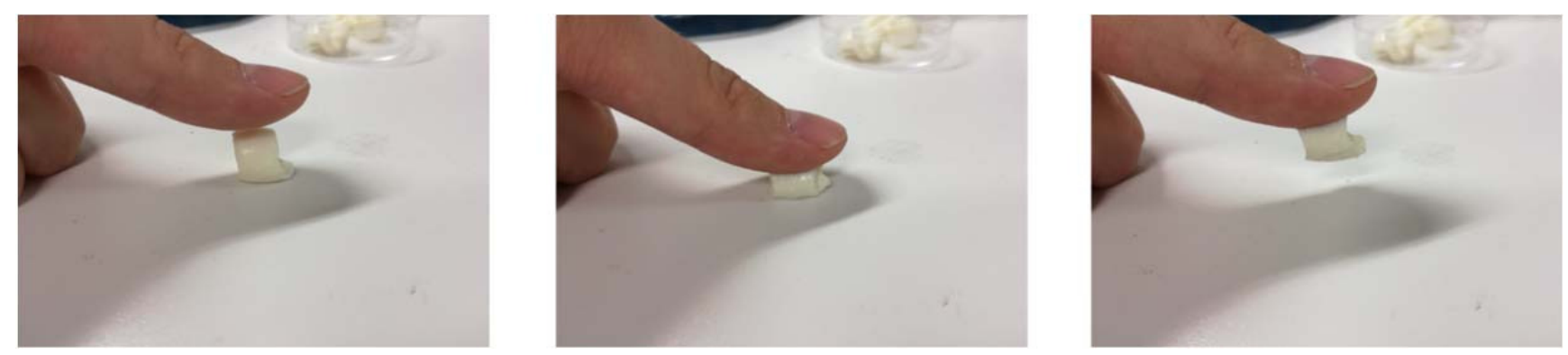

Figure S8. Gentle compression on 2 wt.\% g-CN-AHPA hydrogel and its adhesion to finger. 

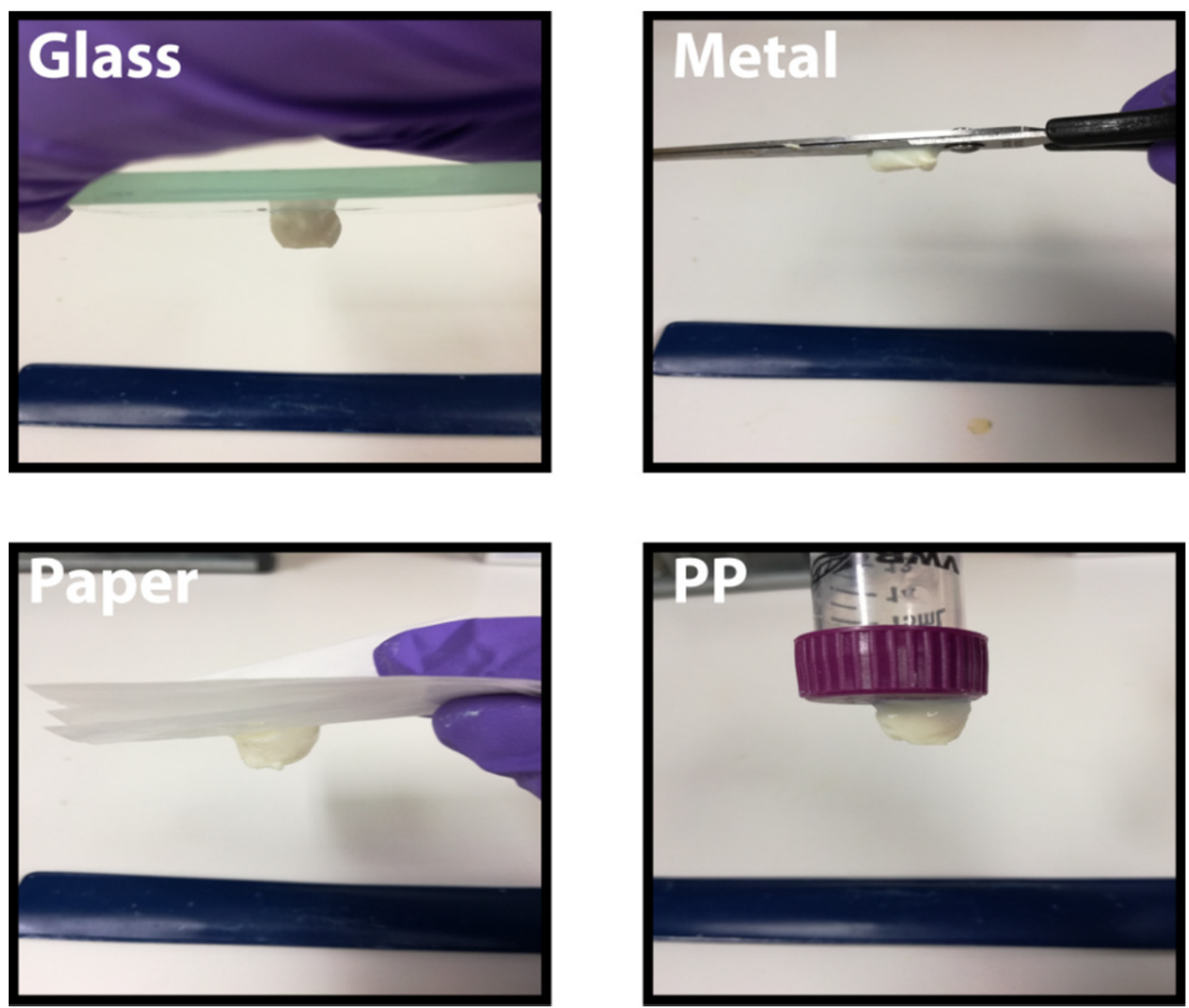

Figure S9. Adhesion of 2 wt.\% g-CN-AHPA hydrogel onto various surfaces. 
a)

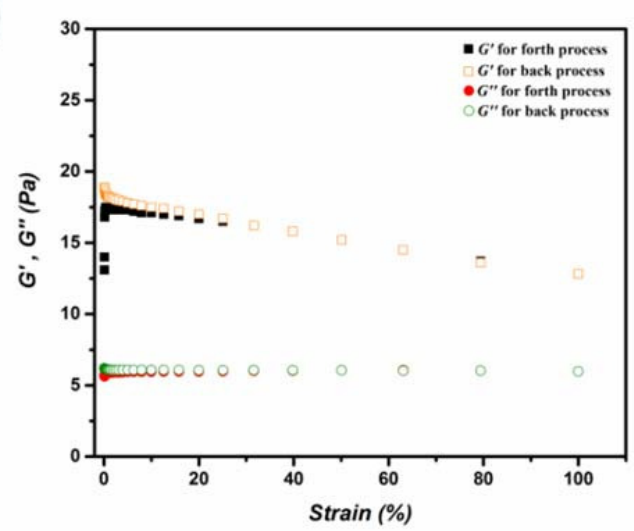

b)

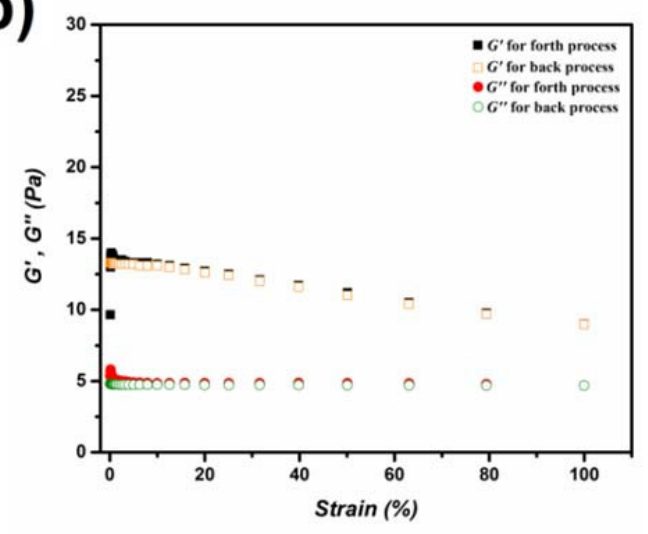

c)

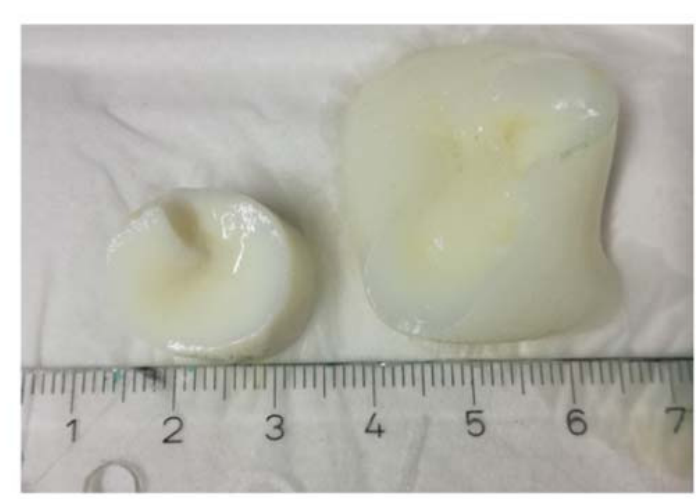

Figure S10. Rheology profiles of 2 wt.\% g-CN-AHPA hydrogel swollen in a) acidic and b) basic media, c) images of swollen hydrogels (left: swollen in acidic media; right: swollen in basic media). 
a)

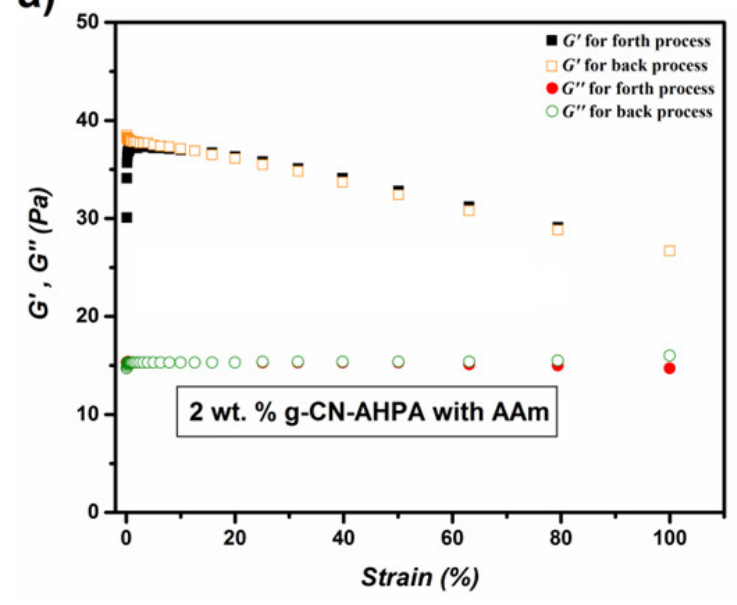

b)

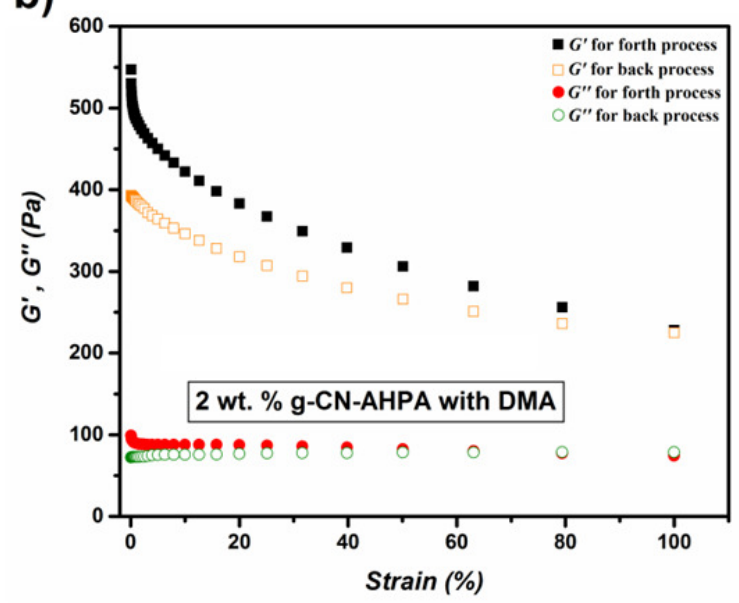

Figure S11. Comparison of storage and loss modulus values of a) 2 wt.\% g-CN-AHPA hydrogel synthesized from AAm, b) 2 wt.\% g-CN-AHPA hydrogel synthesized from DMA against strain (left, $G$ ' values are black and orange squares and $G$ ' values are red and green circles, back (open) and forth (filled) process) and frequency (right).

a)

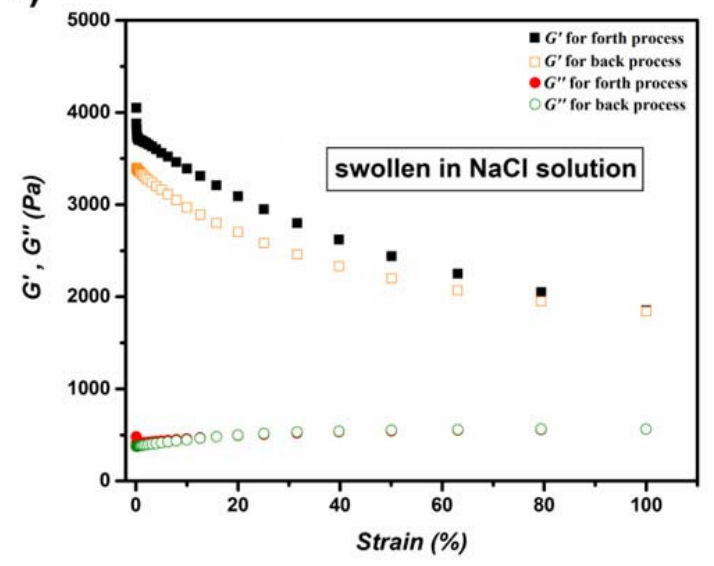

b)

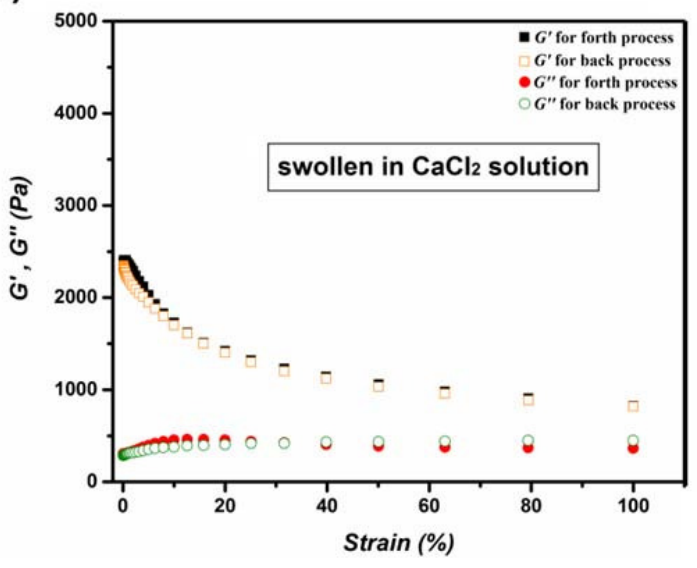

Figure S12. Comparison of storage and loss modulus values of a) 2 wt.\% g-CN-AHPA hydrogel swollen in $\mathrm{NaCl}$ solution $(0.2 \mathrm{M})$ and b) 2 wt. \% g-CN-AHPA hydrogel swollen in $\mathrm{CaCl}_{2}(0.2 \mathrm{M})$ solution against strain (left, $G^{\prime}$ values are black and orange squares and $G^{\prime}$, values are red and green circles, back (open) and forth (filled) process) and frequency (right). 
Table S5. Zeta potential measurements of aqueous g-CN-AHPA dispersions under different conditions.

\begin{tabular}{|l|c|}
\hline \multicolumn{1}{|c|}{ Sample type } & ${\text { Surface Zeta Potential }(\mathbf{m V})^{\mathbf{a}}}^{\mathbf{2}}$ \\
\hline g-CN-AHPA (neutral $\mathbf{p H})$ & -50.5 \\
\hline g-CN-AHPA (at $\mathbf{p H}=\mathbf{4})$ & -13.6 \\
\hline g-CN-AHPA (at $\mathbf{p H}=\mathbf{1 0})$ & -52.7 \\
\hline g-CN-AHPA + NaCl & -33.5 \\
\hline g-CN-AHPA + $\mathbf{C a C l}_{2}$ & -27.6 \\
\hline
\end{tabular}

${ }^{\mathrm{a}}$ Obtained by water dispersion of particles

\section{Supporting Videos}

Video S1. Rotational compression of 2 wt.\% g-CN-AHPA hydrogel via rheometer, its alignment and after compression.

Video S2. Compression of non-flat cut 2 wt.\% g-CN-AHPA hydrogel via compression instrument with $800 \mathrm{~N}$, its transition to paste form upon compression and reshaping after compression.

Video S3. Performing cyclic compression of non-flat cut 2 wt.\% g-CN-AHPA hydrogel (4 cycles recorded on video, 50 cycles performed overall).

Video S4. Repetitive compression with razor blade on 2 wt.\% g-CN-AHPA hydrogel.

Video S5. Circular 2 wt.\% g-CN-AHPA hydrogel elongation and knotting around finger.

Video S6. Smashing 2 wt.\% g-CN-AHPA hydrogel with $3 \mathrm{~kg}$ hammer.

\section{References}

1. Shalom, M.; Inal, S.; Fettkenhauer, C.; Neher, D.; Antonietti, M., J. Am. Chem. Soc. 2013, 135 (19), 7118-21.

2. $\quad$ Kumru, B.; Antonietti, M.; Schmidt, B., Langmuir 2017, 33 (38), 9897-9906.

3. Deng, C.; Cui, Y.; Zhao, T.; Tan, M.; Huang, H.; Guo, M., RSC Adv. 2014, 4 (46), 24095-24102.

4. Huang, T.; Xu, H. G.; Jiao, K. X.; Zhu, L. P.; Brown, H. R.; Wang, H. L., Adv. Mater. 2007, 19 (12), 1622-1626.

5. $\quad$ Wang, L.; Shan, G.; Pan, P., Soft Matter 2014, 10 (21), 3850-6.

6. Bai, T.; Zhang, P.; Han, Y.; Liu, Y.; Liu, W.; Zhao, X.; Lu, W., Soft Matter 2011, 7 (6).

7. Jing, M.; Fu, Y.; Fei, X.; Tian, J.; Zhi, H.; Zhang, H.; Xu, L.; Wang, X.; Wang, Y., Polym. Chem. 2017, 8 (23), 3553-3559. 